\title{
LE TIPOLOGIE DELLE POLITICHE PUBBLICHE: UNA STRADA SENZA USCITA?
}

\author{
di Giliberto Capano
}

\section{Introduzione}

Come è stato recentemente sottolineato, l'analisi delle politiche pubbliche costituisce, insieme al filone della political economy, «il campo d'indagine che ha registrato l'espansione forse più impetuosa nelle scienze sociali dell'ultimo ventennio» (Ferrera 1989, 241). Questo grande sviluppo dell'analisi delle politiche pubbliche pone una serie di problemi concernenti, oltre che il rapporto con la scienza della politica nel suo complesso, gli stessi assetti interni di questo campo di studi. Questioni che nascono soprattutto dal carattere intrinsecamente interdisciplinare dell'analisi delle politiche pubbliche, oltre che dalla problematicità dell'unità di analisi ${ }^{1}$. Nel coacervo di questioni teorico-metodologiche che sorgono, nel momento in cui si intende porre al centro della ricerca empirica la policy, una di quelle più intriganti è costituita dal problema della classificazione delle politiche pubbliche. D'altra parte, il trattamento classificatorio dei concetti occupa un posto fondamentale nel processo di ricerca, costituendo altresì una condizione necessaria, pur se non sufficiente, per procedere in modo adeguato nell'analisi empirica e teorica del fenomeno politico (McKinney 1966, 1970; Tiryakian 1968; Sartori 1979; Oppenheim 1975; Marradi 1990). La questione assume, peraltro, una rilevanza assoluta-

Ringrazio Marco Giuliani, Anna Minelli e Angelo Panebianco che hanno letto e discusso con me una prima stesura di questo articolo, contribuendo a chiarire alcuni passaggi nodali con $i$ loro puntuali suggerimenti; allo stesso modo ringrazio $i$ due anonimi referees che con i loro rilievi ne hanno stimolato la revisione.

${ }^{1}$ Sulle caratteristiche relative ai diversi approcci e sulle diverse matrici disciplinari dell'analisi delle politiche pubbliche risulta particolarmente utile, per il suo impianto ordinatore e la chiarezza espositiva, il contributo di Regonini (1989). Sulle problematiche connesse alla definizione del concetto di policy si veda oltre, n. 20.

RIVISTA ITALIANA DI SCIENZA POLITICA / a. XXIII, n. 3, dicembre 1993 
mente imprescindibile all'interno di un magmatico e frammentato campo di studi, quale è quello dei policy studies, laddove, pur esistendo una notevole tradizione classificatoria - anzi, nella sua espressione più rilevante, tipologica - la diversità delle proposte elaborate (numerose, spesso assolutamente non confrontabili) ha condotto a non soddisfare le attese riposte in tale strumento metodologico per ordinare e guidare la prodigiosa produzione di ricerca (soprattutto a carattere idiografico) sviluppata negli ultimi trent'anni.

In questo articolo miriamo, soprattutto, a presentare una rassegna critica delle principali classificazioni e tipologie delle politiche pubbliche, che sono state elaborate a partire dalla ormai celebre proposta classificatoria avanzata da Theodor Lowi nel 1964. Più specificatamente, l'obiettivo di fondo consiste nel tentare di tirare le somme sullo stato della questione dopo un dibattito che dura da tre decenni, valutando l'adeguatezza, ovvero approfondendo la problematicità, della tipificazione di un concetto sfuggente ed assolutamente non auto-esplicativo come quello di politica pubblica, per cercare di spiegare l'impasse che caratterizza, nel complesso, la tradizione tipologica nei policy studies. In questo senso, anticipiamo subito che non è nostra intenzione approfondire una questione importante che lambisce, ed in alcuni casi coinvolge direttamente, gli sforzi classificatori delle politiche pubbliche: il rapporto tra politics e policy. Ciò si giustifica facilmente se rileviamo come, qualora cedessimo alla tentazione di indulgere su questo argomento, allargheremmo indebitamente la prospettiva analitica fino ad abbracciare il problematico rapporto che sussiste tra scienza politica nel suo complesso e policy studies. Nella nostra analisi, quindi, la concezione del circuito policy-politics che informa le specifiche costruzioni tipologiche verrà considerata solo in relazione alla scelta delle dimensioni su cui vengono costruite le tipologie, poiché è in base alla concezione delle determinanti dei processi politici che i vari studiosi selezionano i criteri fondanti le classificazioni tipologiche.

L'argomento verrà, quindi, trattato selezionando i contributi disponibili sul tema, attraverso l'individuazione dei principali criteri e/o dimensioni su cui sono state costruite le tipologie delle politiche pubbliche; perciò, dopo aver brevemente accennato ai primi tentativi di classificazione, analizzeremo e criticheremo quei filoni della riflessione tipologica che hanno concentrato la loro attenzione sulle dimensioni della coercizione, della 
disaggregabilità e delle caratteristiche sistemiche ${ }^{2}$, restando, però, all'interno della logica classificatoria tradizionale. Nella parte conclusiva dell'esposizione accenneremo, invece, alle problematiche connesse al ruolo ed all'utilizzabilità delle suddette tipologie nell'analisi delle politiche pubbliche, ragionando sulle possibilità di superare l'impasse teorica ed empirica che sembra caratterizzare intrinsecamente $i$ contributi dell'approccio tipologico alle politiche pubbliche ${ }^{3}$.

\section{Le prime proposte di classificazione}

Alcuni cenni su quelle classificazioni tradizionali, fondate su criteri auto-esplicativi e meramente descrittivi, che risultano insoddisfacenti a chi adotti la prospettiva dei policy studies. Classificazioni di questo tipo, usate abitualmente non solo nel linguaggio comune ma anche, spesso, dagli studiosi del fenomeno politico, sono numerose, ma possono essere ricondotte ad alcune categorie generali. Le classificazioni tradizionali, che ricorrono con elevata frequenza nella letteratura politologica, sono quelle fondate su: categorie sostantive (politiche del lavoro, dell'educazione, sanitarie, di welfare, ecc.); categorie basate sul livello di go-

2 Ovviamente, nella nostra ricostruzione non possiamo tenere conto di tutte le proposte di classificazione avanzate negli ultimi trent'anni. Abbiamo, quindi, cercato di raggrupparle a seconda della rilevanza teorica e del riconoscimento, magari anche critico, ottenuto. Sono restate, perciò, escluse classificazioni che, pur formulate all'interno di un dibattito ormai vivace sulla questione, non hanno ricevuto, forse giustamente, un'attenzione significativa. Citiamo, a mo' di esempio, proposte classificatorie come quelle che distinguono tra politiche adattive e di controllo (Eulau e Eyestone 1968); tra politiche di area e segmentate (Froman 1967; 1968); tra politiche che allargano o restringono le alternative di comportamento (Bressers e Honigh 1986).

${ }^{3} \mathrm{Da}$ un punto di vista metodologico, l'analisi delle tipologie che opereremo si atterrà, in gran parte, alla logica classificatoria positivista, laddove la classificazione è un'operazione intellettuale (Marradi 1987), attraverso la quale viene suddivisa in classi l'estensione di un concetto. In questa logica la tipologia è una classificazione che si contraddistingue per il carattere multidimensionale, ovvero per essere costruita su almeno due criteri (o dimensioni del medesimo criterio). L'attribuzione delle classi, come è noto, deve essere esaustiva e mutualmente esclusiva. Il fine di tale trattamento logico dei concetti è quello di costruire strumenti analitici capaci di ordinare la ricerca e l'immagazzinamento dei dati (Sartori 1979; Tiryakian 1968). Rileva sottolineare, però, anche ai fini della nostra trattazione, come la costruzione di tipologie possa uscire dalla logica classificatoria positivista, e quindi dalla cogenza dei requisiti della mutua esclusività ed esaustività. $\grave{E}$ il caso degli idealtipi weberiani, che identificano quadri ideali dei processi che si verificano in un determinato contesto storico-politico, attraverso una costruzione teorica «conseguita mediante l'accentuazione concettuale di determinati elementi della realtà» (Weber 1922a, trad. it. 1980, 107). 
verno (politiche comunitarie, statali, locali, regionali, intergovernative) e categorie ideologiche (politiche liberali, conservatrici, secolari, confessionali, ecc.). Vi possono essere, poi, altre catalogazioni delle policies costituite da categorie fondate sui destinatari (politiche per gli agricoltori, per le minoranze, ecc.); oppure su un criterio storico-temporale (politiche post-belliche; politiche del periodo centrista, ecc.); oppure su giudizi di valore (politiche buone, cattive, pericolose) o, infine, sulle caratteristiche del consenso (politiche consensuali o conflittuali) (Froman 1968).

L'esigenza di elaborare classificazioni delle policies nasce dalla necessità di superare i limiti euristici che le categorie appena elencate pongono allo sviluppo teorico dell'analisi delle politiche, anche in prospettiva comparata. In effetti, sebbene sia stato affermato che alcune di queste categorie potrebbero soddisfare $\mathrm{i}$ requisiti di adeguatezza richiesti ad una classificazione per poter essere vantaggiosamente utilizzata nella ricerca ${ }^{4}$, esse, nel complesso, sono informate da una caratteristica comune che ne inficia la spendibilità a fini euristici. Si tratta dell'eccesso di vicinanza ai dati, di un basso livello di astrazione che rende improponibile la loro utilizzazione per generare ipotesi a medio raggio, ovvero per procedere ad analisi comparate, nella prospettiva di ricerca dei policy studies. Più specificamente, il problema di fondo è quello di superare l'eccesso di descrittività ovvero il rischio di reificazione che è proprio delle classificazioni tradizionali (invero delle vere e proprie catalogazioni descrittive $)^{5}$. È, perciò, proprio per cercare di supplire al deficit, connaturato a tali classificazioni descrittive, rispetto alla generazione di ipotesi sulle relazioni causali, che emerge tra gli studiosi, soprattutto statunitensi, l'esigenza di formulare concettualizzazioni che consentano maggiori possibilità euristiche all'analisi dei processi decisionali pubblici. L'elemento di fondo che spinge verso questi tentativi consiste nell'ipotesi che «esistano fattori trasversali capaci di dare una struttura simile a processi decisionali che pure si dispiegano in settori diversi» (Regonini 1989, 510).

4 Froman ritiene, in base ad una serie di parametri (inclusività, mutua esclusività, validità, attendibilità, livello di misurazione, possibilità di operazionalizzazione, possibilità di correlazione con altri fenomeni) che siano abbastanza soddisfacenti le classificazioni fondate sui criteri: storico-temporale, ideologico e del consenso (Froman 1968, 46-48).

5 Fanno eccezione, a questo eccesso di descrittività, le classificazioni fondate su criteri valoriali, le quali si caratterizzano, piuttosto, per un eccesso di soggettivismo unito ad un'elevata dose di prescrittività. 
Gli sforzi in questo senso cominciano a prendere corpo a partire dagli anni sessanta anche se, precedentemente, vi erano già stati tentativi, seppur in campi di indagine differenti, di inferire determinati comportamenti politici dalle caratteristiche delle issues trattate. A questo proposito, basti citare la classificazione, utilizzata nell'analisi del comportamento dei partiti statunitensi nelle campagne elettorali, laddove si distingueva tra questioni «concrete» e «generali» rispetto alle quali si ipotizzava variassero le preferenze partitiche (Berelson, Lazarsfeld e McPhee 1951); oppure quella di Huntington (1961) che distingueva tra questioni strategiche e strutturali, nell'ambito dei processi decisionali del settore militare.

Per quanto riguarda la prospettiva analitica che pone al centro dell'indagine la policy, il primo vero contributo di tipo classificatorio è quello avanzato da Lowi nell'ormai classico lavoro pubblicato nel 1964 sulla rivista «World Politics» (1964a). Si deve sottolineare come quella prima proposta classificatoria, certo una pietra miliare per la prospettiva dei policy studies, tenda a differenziarsi in alcuni aspetti rilevanti dai contributi successivi dello stesso autore, laddove questi verrà a proporre una vera e propria tipologia. E le differenze rilevabili sono significative per il nostro percorso critico. Nel suo primo contributo, Lowi prende spunto dalla recensione di un volume di Bauer et al. (1963) sulla politica statunitense del commercio estero, per affrontare l'analisi critica degli approcci teorici allo studio del potere e del policy-making. Lowi, individuando tre principali modelli teorici (quello pluralista, quello elitista-stratificazionista e quello della frammentazione ovvero delle «mutue non-interferenze» di Schattschneider), ne critica la valenza assolutamente auto-esplicativa ed auto-referenziale e l'incapacità intrinseca a fornire schemi adeguati per l'analisi empirica, soprattutto a causa della pretesa, insita in ognuno degli approcci teorici tradizionali, che esista un'unica struttura di potere ed un'unica fenomenologia del processo decisionale. L'obiettivo è, quindi, quello di costruire uno schema classificatorio capace di superare le aporie proprie degli schemi tradizionali e, in particolare, di «cumulare, comparare e mettere a confronto scoperte differenti» (Lowi 1964a, 688).

Il quadro concettuale sul quale poggia lo schema classificatorio di Lowi si basa sui seguenti elementi: a) le relazioni tra individui, comprese quelle politiche, sono fondate sulle aspettative di poter ottenere qualcosa interagendo; $b$ ) in politica le 
aspettative sono determinate dalle decisioni governative; c) una relazione politica è determinata dalla policy in gioco, quindi, in ogni tipo di policy esiste uno specifico tipo di relazione politica. Su queste basi si può procedere all'individuazione dei tipi di politiche pubbliche, operazione che necessita, comunque, di un'ulteriore specificazione analitica, conseguenza della logica che lega $\mathrm{i}$ tre assunti sopra esposti. Si devono, cioè, definire le policies «nei termini del loro impatto o del loro impatto atteso sulla società» (Lowi 1964a, 689). In base a questa definizione, Lowi individua tre principali categorie di politiche pubbliche: quella distributiva, quella regolativa e quella redistributiva. Questi tipi sono «storicamente» e «funzionalmente» definiti ed identificano determinate arene di potere, ciascuna caratterizzata da una propria specificità per quanto concerne la struttura di potere, il processo decisionale, le élites ed i gruppi presenti e le loro relazioni.

Le politiche distributive si caratterizzano per attribuire un beneficio immediato a destinatari visibili, occultando i costi, e per essere facilmente disaggregabili (senza tener conto della limitatezza delle risorse); sono, in sostanza, le politiche in cui non vi è mai confronto tra chi vince e chi perde. Questo tipo di politica vede la presenza di un gran numero di gruppi di interesse, in un contesto relazionale caratterizzato da una bassa, se non inesistente, conflittualità, dovuta non tanto al compromesso e alla negoziazione quanto, piuttosto, all'assoluta mancanza di confrontabilità tra gli interessi rappresentati. Proprio questa caratteristica consente quel tipo di interazione politica definita mutual non interference, logrolling, pork-barrel. La sede privilegiata è quella di organismi ristretti come le commissioni parlamentari oppure le agenzie amministrative.

Le politiche regolative si differenziano da quelle distributive per non poter essere disaggregate all'infinito. Ciò poiché esse implicano, secondo Lowi, un impatto chiaramente indirizzato ad aumentare i costi, ovvero a ridurre le alternative al comportamento individuale. Inoltre «le politiche regolative si distinguono da quelle distributive per il fatto che, nel breve periodo, esse implicano una scelta diretta tra chi riceverà una concessione e chi, invece, una privazione» (Lowi 1964a, 694). La possibilità di questa scelta comporta il formarsi di due coalizioni contrapposte, all'interno delle quali si innescano interazioni impensabili in una politica distributiva laddove, come abbiamo visto, si raggiunge un equilibrio fattuale tra le diverse, e non confron- 
tabili, scale di preferenza dei partecipanti. Per queste sue caratteristiche, l'arena regolativa, la cui sede privilegiata è quella assembleare, si caratterizza per una particolare instabilità delle coalizioni ed un elevato potenziale di conflittualità.

Le politiche redistributive si differenziano da quelle regolative per la maggiore ampiezza dell'impatto che esse implicano, allargando il proprio spettro di riferimento alle classi sociali, e per un potenziale ancora minore di disaggregabilità. Questo tipo di politica si caratterizza per essere trattata a livello di esecutivo, coinvolgendo le grandi associazioni di interesse in un contesto relazionale istituzionalizzato, stabile e fortemente ideologizzato.

Abbiamo dedicato uno spazio superiore a quello realmente necessario, visto che essa è ben nota agli studiosi, alla prima formulazione dello schema classificatorio lowiano per dare maggior risalto e comprensibilità ad alcune considerazioni critiche su significative aporie metodologiche che ne caratterizzano l'elaborazione (sulle questioni di sostanza rimandiamo al paragrafo successivo). La prima riflessione che merita avanzare concerne la difficoltà ad individuare il criterio, o i criteri, in base al quale vengono differenziati i tre tipi di policy. Dall'enfasi con la quale viene sottolineato, sembrerebbe che il criterio fondante della classificazione sia costituito dall'impatto atteso dell'attività di governo sulla società (dalla società?). D'altra parte, alcuni autori hanno individuato la disaggregabilità della policy - ovvero la frammentazione della politica in micro-decisioni individuali ed individualizzanti - come dimensione fondamentale della prima proposta classificatoria di Lowi (Kjellberg 1977; Uslaner 1985; Regonini 1985; Giuliani 1992). Il problema di fondo, a questo proposito, è che la disaggregabilità della policy, nella logica argomentativa dell'autore statunitense, non viene direttamente individuata come il criterio costitutivo della sua classificazione, bensì come un elemento esplicativo delle caratteristiche dei singoli tipi, insieme a quello della ripartizione dei costi e dei benefici. D'altra parte, senza ricorrere alla disaggregabilità, e alla ripartizione - per come percepita dai destinatari - dei benefici e dei costi, il criterio cui Lowi attribuisce esplicitamente la capacità di distinguere differenti tipi di politiche, l'impatto o l'impatto atteso sulla società, non è sufficiente ad individuare classi mutualmente esclusive, se non ricorrendo ad una specificazione del concetto che di fatto porta Lowi ad utilizzare, appunto, tre differenti criteri analitici: la disaggregabilità, la ripar- 
tizione dei costi e dei benefici e l'impatto della decisione ${ }^{6}$. In effetti, nello schema di Lowi, la differenza tra il tipo distributivo e quello regolativo sta nelle diverse caratteristiche rispetto al potenziale disaggregativo (pressoché infinito per il primo, limitato per il secondo) e nella ripartizione dei costi e dei benefici e non nell'impatto della decisione a livello di società, poiché entrambi i tipi sono caratterizzati da un «impatto specifico ed individuale» (Lowi 1964a, 690). Allo stesso modo, la differenza tra le politiche regolative e quelle redistributive sta proprio «nella natura dell'impatto»: nelle politiche redistributive «le categorie di impatto sono più larghe, avvicinandosi alle classi sociali» (Lowi 1964a, 691). Per contro, politiche regolative e redistributive sono ritenute omogenee per quanto riguarda la disaggregabilità e la suddivisione dei costi e dei benefici.

Nella sostanza, quindi, questo primo contributo lowiano soffre di una profonda ambiguità per quanto concerne il criterio (o i criteri?) sui quali egli fonda la sua proposta classificatoria (o tipologica?). Attenendosi alla logica classificatoria, il criterio dell'impatto non è un buon criterio, poiché individuerebbe, se applicato correttamente, due tipi e non tre; gli stessi risultati si otterrebbero utilizzando il criterio della disaggregabilità e quello della ripartizione dei costi, per come utilizzato da Lowi ${ }^{7}$. D'altra parte, su questo punto, si deve sottolineare come

6 È interessante sottolineare, a questo proposito, l'ambiguità con cui viene trattato il concetto di impatto. Lowi utilizza questo termine in due accezioni: solitamente riferendosi all'ampiezza dei settori sociali, o degli aggregati sociali coinvolti dall'output (successivamente utilizzerà la definizione di ambito della coercizione), mentre a volte, invece, il termine impatto viene adoperato nel senso di contenuto e obiettivi dell'output decisionale (Lowi 1964a, 690). Smith $(1969 ; 1975)$ utilizza intercambiabilmente il termine di impatto e quello di outcome, in una interpretazione, peraltro, più convincente, anche se non esaustiva. È evidente, infatti, che, se l'elemento caratterizzante una policy è l'aspettativa degli attori - fondata sull'esperienza passata - rispetto al futuro della politica pubblica, allora questa aspettativa può riguardare sia le proposte di policy, sia l'output sia l'outcome. Insomma, il termine «impatto» sembra quantomeno improprio nel significato primario in cui lo utilizza solitamente Lowi poiché, nella logica della sua argomentazione, le attese sembrano concernere soprattutto gli esiti della formulazione del processo decisionale. Sul punto si veda anche: Greenberg et al. (1977, 1534-36); Sheffer (1977, 537 ss.); per una concezione dell'impatto omogenea a quella utilizzata da Lowi si veda Rakoff e Schaefer (1970); per contro, oltre che a Smith, rimandiamo a Rose (1969) per una concezione che usa il termine impatto come sinonimo di outcome.

7 Lowi adopera in modo molto semplificato il criterio dei costi e dei benefici: nelle politiche distributive, $i$ costi sono nascosti ed i benefici trasversali (poiché essi riguardano non solo i vincitori, ma anche i soggetti più influenti tra i perdenti); nelle politiche redistributive e regolative, essi sono concepiti come visibili e a somma-zero (da una parte $i$ vincenti, dall'altra $i$ perdenti). Come vedremo, Wilson ragionerà in modo più raffinato intorno all'applicabilità di tale criterio. 
l'ambiguità della prima proposta classificatoria di Lowi sia sostanzialmente speculare all'ambiguità costitutiva del fenomeno politica pubblica. Tale ambiguità fenomenologica è estremamente rilevante se si cerca di classificare il contenuto delle politiche pubbliche guardando al loro percorso evolutivo e sequenziale. Si riscontra, allora, la modificazione del contenuto delle politiche (la politics) passando da una fase all'altra del policymaking. Lowi prende progressivamente coscienza di tale antinomia, giungendo, come vedremo, a ridurre di molto la definizione operativa di policy.

Le tipologie fondate sulle dimensioni della coercizione: da Lowi a Lowi?

«La coercizione è a livello macro-sociale ciò che il potere è a livello micro o comportamentale» (Lowi 1970, 314). Questa affermazione costituisce l'assunto di fondo in base al quale Lowi imposta la costruzione della proposta tipologica che, rimodellando la classificazione precedente, diviene un punto di riferimento, anche in senso fortemente critico, per gli studiosi dei processi decisionali. La logica conseguenza di un simile cambiamento di prospettiva - il passaggio da un'ottica micro, fondata sull'analisi delle interazioni individuali, ad un'analisi macro, centrata sul ruolo delle variabili strutturali - consiste nella riformulazione del concetto di policy, di cui viene enfatizzata l'extrema ratio, in quanto «dopo tutto, la policy non è che deliberata coercizione, cioè un insieme di statuizioni finalizzate a fissare i propositi, i mezzi, i soggetti e gli oggetti della coercizione» (Lowi 1970, 315). Nella sostanza, optare per questa prospettiva significa porre al centro dell'attenzione analitica l'attività di governo $\mathrm{e}$, più specificamente, il fenomeno più importante relativo al governo: il fatto che l'attività di governo si fonda sulla coercizione. Quindi, «i differenti modi di usare la coercizione provvedono a fornire un insieme di parametri, un contesto, dentro cui la politica (politics) ha luogo» (Lowi 1972, 299).

La fig. 1 riporta la rappresentazione grafica della tipologia che Lowi costruisce individuando due dimensioni della coercizione: il campo di applicazione (che può essere limitato alla condotta individuale dei soggetti oppure allargato al contesto ambientale nel quale i soggetti si muovono) e la probabilità della sua applicazione (che può essere immediata se la sanzione - 
Ambito di applicazione della coercizione

\begin{tabular}{|c|c|c|c|}
\hline & & $\begin{array}{l}\text { Condotta } \\
\text { individuale }\end{array}$ & $\begin{array}{c}\text { Ambiente } \\
\text { della condotta }\end{array}$ \\
\hline \multirow{2}{*}{$\begin{array}{l}\text { Probabilità } \\
\text { della } \\
\text { coercizione }\end{array}$} & Remota & Politica distributiva & Politica costituente \\
\hline & Immediata & Politica regolativa & Politica redistributiva \\
\hline
\end{tabular}

FIG. 1. Tipi di coercizione e tipi di policy (Lowi 1970)

intesa anche come costo - incide direttamente e visibilmente sui destinatari della politica ovvero remota se la sanzione è assente $o$ indiretta).

L'incrocio delle due dimensioni della coercizione, individuate come rilevanti da Lowi, consente di allocare, all'interno dello schema tipologico in tal modo delineato, la tripartizione delle politiche precedentemente elaborata (arricchendo ogni tipo di ulteriori specificazioni) ed aggiungendo, poi, un quarto tipo di politica pubblica, quella costitutiva (o costituente, o costituzionale, o istituzionale $)^{8}$.

${ }^{8}$ Quella delle politiche costituenti rappresenta la categoria meno approfondita ed analizzata da parte di Lowi e dei suoi epigoni. Probabilmente hanno ragione coloro $\mathrm{i}$ quali ritengono che questo quarto tipo di politica pubblica sia stato individuato, soprattutto, per dare maggiore omogeneità ed eleganza alla quadripartizione tipologica, per riempire, insomma, la quarta casella. Non è un caso, peraltro, che in tutte le altre classificazioni tipologiche che non seguono la lezione di Lowi, la quarta cella sia riempita in modo differente, e comunque egualmente discutibile. La forzatura compiuta da Lowi sta, soprattutto, nel fatto che, denominando costituente la politica inclusa nella quarta cella a sua disposizione, egli estremizza alcune dinamiche proprie delle politiche che hanno come oggetto le regole del gioco [tale è il contenuto di tali politiche anche secondo lo stesso Lowi $(1985,75)$ ]: soprattutto il fatto che, spesso, tali processi decisionali tendano ad assumere connotati fortemente distributivi. Se ciò fosse completamente attendibile, come si potrebbe assegnare a tale categoria quelle politiche di riforma amministrativa, oppure quelle politiche organizzative che, in alcuni paesi occidentali, non sembrano essere caratterizzate in modo determinante da elementi distributivi? Invero, il tipo costituente è decisamente fuori posto nella quadripartizione di Lowi. Tale politica sembra contraddistinguersi, soprattutto, per la sua afferenza sostanziale, per il contenuto di cui essa tratta (le regole e le procedure delle altre politiche); è evidente, perciò, che essa può presentare elementi sia regolativi, che redistributivi, che distributivi. Che, nella realtà, essa possa assumere connotati soprattutto distributivi dipende dalle caratteristiche degli attori coinvolti (solitamente le élites politiche ed amministrative) i quali, però, non necessariamente debbono sviluppare uno stile decisionale consensuale e coo- 
L'individuazione delle dimensioni della coercizione come criterio fondante la delimitazione di differenti tipi di policy costituisce una precisa scelta di prospettiva, che implica la focalizzazione dell'azione (o, meglio, dell'intenzione) autoritativa dei decisori pubblici come momento centrale del decorso di una politica pubblica. Con questa scelta, Lowi abbandona l'ambiguità riscontrabile nei primi lavori sulla classificazione dei tipi di politica (Lowi 1964a; 1964b; 1966; 1967), in cui sconta l'indecisione teorica rispetto al ruolo autoritativo dello Stato - considerato dallo stesso Lowi come centrale per una teoria del fenomeno politico - e l'eccesso di enfatizzazione delle aspettative degli attori partecipanti. Una simile scelta, però, se risulta funzionale all'eleganza dell'elaborazione teorica, non contribuisce ad eliminare le problematiche teoriche ed empiriche connesse al suo contributo tipologico.

Restano immutate, rispetto alla tripartizione precedente, questioni come la non mutua esclusività delle categorie, la problematica operazionalizzazione delle caratteristiche proprie dei tipi di politica individuati e la forte tendenza, insita nella logica delle dimensioni scelte per la costruzione della tipologia, a ricadere nell'ambito di classificazioni fondate sull'afferenza formale ed istituzionale. In relazione alla questione della mutua esclusività, lo stesso Lowi è consapevole del fatto che, almeno sul lungo periodo, le politiche possono assumere tendenze differenti rispetto a quelle originarie (Lowi 1964a, 690). Tale tendenza fattuale delle politiche costituisce un vincolo che, come è stato sottolineato ripetutamente (Salisbury 1968; Wilson 1973; Hofferbert 1974; Greenberg et al. 1977; Kjellberg 1977; Hayes 1978; Richardson et al. 1982; Spitzer 1983a; 1983b; 1987; Tatalovich e Dynes 1984; Uslaner 1985), rende assai difficoltosa la soddisfazione del requisito della mutua esclusività delle classi nella classificazione dei dati reali, tranne che nei casi estremi (Wilson 1973, 328). Il problema, lo ribadiamo, dipende dall'utilizzo che viene fatto delle categorie tipologiche. Se esse vengono adoperate per classificare processi dinamici, evidentemente risultano inadatte; dall'altra parte, anche decidendo di assumere uno specifico momento della policy come la più rappresentativa (l'impatto sulla società, o la decisione formalizzata), resta il problema di individuare le caratteristiche operative della coercizio- 
ne (esse esistono oggettivamente oppure sono delineabili facendo riferimento alla percezione degli attori?)

Per quanto riguarda le due altre aporie, sottolineiamo come: a) rispetto al rischio di una riduzione alla mera afferenza formale delle politiche pubbliche, lo stesso Lowi sostenga, già nelle sue prime riflessioni, una posizione fortemente ambigua, laddove individua una stretta correlazione tra strutture amministrative e tipi di politica (Lowi 1970, 323), che favorisce le interpretazioni in tal senso della sua classificazione (Hill e Plumlee 1984; Dente 1990); b) il modello tipologico sia difficilmente testabile, poiché l'operazionalizzazione dei concetti di base (coercizione, aspettative degli attori, definizione di policy, impatto) obbliga il ricercatore ad un eccesso di assunzioni ed interpretazioni soggettive (Greenberg et al. 1977) che diluiscono la cogenza del modello di partenza.

Di fronte a tali limiti, tre soluzioni sarebbero possibili per mantenere l'utilizzabilità della tipologia fondata sulla coercizione, senza abbandonarne la logica costitutiva e restando nell'ambito della tradizione tipologica positivista. La prima è quella di classificare le politiche pubbliche in base al criterio, comunque difficile da fondare empiricamente, della «prevalenza» di determinate caratteristiche rispetto alle altre; ed è questa la strada che Lowi sembra inizialmente privilegiare e che, solitamente, e giudiziosamente, viene percorsa, anche a meri fini descrittivi, dagli analisti delle politiche. Il criterio della prevalenza, però, se può soddisfare il problema della mutua esclusività, anche in modo inelegante, certo non aiuta affatto rispetto alle altre due aporie.

La seconda strada consiste nel considerare le due dimensioni della coercizione che fondano la tipologia lowiana non come dicotomiche, bensì come due continua, in modo tale da trattare le politiche da classificare non più in termini discreti bensì di grado (Spitzer 1983a; 1983b; 1987; Heckathorn e Maser 1990). Su questa via, accanto ai tipi puri (che sono raramente riscontrabili nella realtà), si possono collocare dei tipi definibili come «misti» che costituiscono la gran parte dei casi reali. Questo tentativo di riconsiderare e rielaborare la tipologia di Lowi risulta, però, soffrire delle medesime problematiche connesse all'operazionalizzazione (come misurare la probabilità ovvero l'ambito di applicazione della coercizione?) e, in realtà, costituisce una riproposizione più complessa ed articolata del metodo della prevalenza. Che differenza vi è tra classificare una politica, 
che ha connotati anche regolativi, come prevalentemente distributiva rispetto al classificarla come «distributiva mista» (poiché ha un livello di coercizione meno remoto che l'avvicina al tipo regolativo)? Questa soluzione appare non solo inelegante (Kellow 1988) rispetto alla parsimonia dell'elaborazione originaria ma anche, e soprattutto, del tutto inutile a risolvere i limiti di cui stiamo discutendo.

La verità, e siamo alla terza possibilità di risolvere le aporie delle tipologie basate sulla coercizione, tentando di salvaguardarne l'efficacia euristica ed il rispetto della logica classificatoria, è che l'individuazione della coercizione come dimensione fondante la tipologia impone la scelta di una determinata prospettiva d'analisi, tesa a restringere decisamente la delimitazione del concetto di policy. Ed è questa la strada che Lowi - risolvendo le contraddizioni di fondo che hanno caratterizzato $i$ suoi contributi sulle politiche pubbliche, e riprendendo alcuni spunti del suo The End of Liberalism (1969, 88 ss.) - sembra aver intrapreso recentemente. Ė una prospettiva «istituzionalista» che caratterizza questa nuova versione della classificazione tipologica, laddove essa diviene da una parte uno schema per individuare diversi regimi politici (Lowi 1985, 1988) dall'altra la tipificazione di un concetto, quello di policy, assolutamente privato di tutti quegli elementi della politica che non siano strettamente connessi agli interessi delle istituzioni.

In questa opera di riduzione e formalizzazione del concetto, la policy diventa «una regola, formulata da qualche autorità governativa, che esprime un'intenzione di influenzare il comporta-

9 Spitzer, ad esempio, è ben cosciente che l'introdurre differenze di grado nelle categorie nominali di Lowi comporta problemi, ancora irrisolti, di misurazione della coercizione. D'altra parte, nell'applicazione empirica di tale tipologia (Spitzer 1983a; 1983b), egli utilizza, come parametri di attribuzione delle politiche alle varie categorie, la natura dell'issue e le decisioni prese precedentemente nell'ambito di policy dato. Gli indicatori adoperati sono quantomeno discutibili: affermare che una policy o un'issue è per sua natura mista, creando, in realtà, una specie di «tipo puro misto», non fa altro che aggiungere categoria a categoria; ritenere che le decisioni passate possano creare tipi misti, non ci assicura che la medesima policy mantenga le stesse caratteristiche nel futuro (per quanto, è chiaro, la legislazione vigente costituisce un vincolo, non assolutamente coercitivo, per il decorso di una policy). Peraltro, l'applicazione che Spitzer fa della sua proposta tipologica in relazione alle politiche regolative (Spitzer 1987) - distinguendo tra vecchia regolazione (economica) e nuova regolazione (sociale), e di fatto creando 4 tipi di regolazione (regolazione sociale pura e mista; regolazione economica pura e mista) - ci dimostra chiaramente come la sua concezione delle caratteristiche determinanti le categorie tipologiche sia strettamente correlata, troppo diciamo noi, alla presunzione di una supposta natura intrinseca dell'issue. 
mento individuale o collettivo dei cittadini attraverso l'uso di sanzioni negative e/o positive» (Lowi 1985, 70). Imboccando questa prospettiva, Lowi trasforma una dimensione della sua tipologia, sostituendo la probabilità della coercizione con la forma giuridica attraverso la quale vengono espresse le intenzioni dei decisori; questa dimensione viene dicotomizzata, utilizzando la distinzione delle regole giuridiche operata da Hart (1961) tra regole primarie (che impongono sanzioni ed obbligazioni) e regole secondarie (che conferiscono privilegi o poteri). L'obiettivo di tale reimpostazione in termini giuridico-formali (laddove la norma giuridica è l'espressione dei rapporti tra cittadini ed attività autoritative delle istituzioni) è proprio quello di eliminare le problematiche che derivano dall'includere, all'interno della definizione di policy, elementi relativi alle aspettative degli interessi sociali e politici; con tale riduzione viene meno anche il problema della «prevalenza» di un aspetto rispetto ad un altro, poiché la classificazione ha come referente l'ordinamento giuridico, o altre espressioni formalizzate della decisione.

Al tempo stesso viene, sempre secondo Lowi, eliminata l'ambiguità che sussiste in relazione all'elemento temporale, poiché se la classificazione ha come oggetto le forme giuridico-normative attraverso cui la decisione è stata formalizzata, la sua collocazione in una delle quattro classi non può essere messa in discussione nel caso che, dieci anni dopo la sua statuizione, essa venga ad assumere connotati differenti, riconducibili ad un diverso tipo. Riducendo all'aspetto coercitivo le interazioni politiche, e privilegiandone la dimensione istituzionale, in quanto «è la coercizione del governo che rende la partecipazione inevitabile» (Lowi 1988, 727), Lowi ritiene di purificare al massimo la definizione di policy, in modo tale da poter meglio fondare la sua prospettiva teorica (policy determines politics), senza lasciare adito a disparate e soggettive interpretazioni. Il nocciolo duro dell'analisi lowiana, che costituisce il momento terminale di una parabola che progressivamente riduce la problematicità e l'estensione del concetto di politica pubblica, è una classificazione fondata sull'esigenza che «la suddivisione in categorie della politica pubblica, che è la variabile indipendente, sia intesa come uno sforzo per catturare le intenzioni dei decisori così come espresse dal linguaggio formalmente stabilito del governo» (Lowi 1985, 75).

Una simile riduzione (del concetto e del fenomeno di poli$c y)$, al di là dei problemi connessi con la capacità di cogliere la 
problematicità fenomenologica delle politiche pubbliche, non risolve, inoltre, la questione della mutua esclusività delle classi, poiché, solitamente, $i$ testi normativi presentano notevole ambiguità rispetto agli obiettivi e agli strumenti da utilizzare. Riecheggia, nella locuzione «afferrare le intenzioni dei decisori», la questione dell'«interpretazione della volontà del legislatore» che è tanto rilevante per $\mathrm{i}$ cultori delle discipline gius-pubblicistiche, quanto fuorviante per gli studiosi del fenomeno politico: è davanti alla questione dell'interpretazione del diritto che le strade della scienza giuridica e della scienza della politica si dividono nel metodo e negli obiettivi euristici. In questa prospettiva, la riduzione della policy a rule, se nasce dal tentativo di trovare un equilibrio tra la prospettiva istituzionalista e quella behaviorista (Calise 1987), tende, a causa dell'enfatizzazione dell'elemento giuridico-normativo, a riproporre una visione tradizionale delle istituzioni, di fatto appiattite sull'elemento giuridico-formale.

\section{Le tipologie fondate sulla disaggregabilità delle politiche}

Come abbiamo sottolineato, la dimensione della disaggregabilità delle politiche assume un ruolo ambiguo nelle tipologie fondate sul criterio della coercizione, soprattutto perché l'ansia di salvaguardare il fine teorico-esplicativo che sta alla base di quella costruzione tipologica conduce a sottostimare le possibilità analitiche e classificatorie proprie del potenziale disaggregativo di una politica pubblica. L'approfondimento di questo elemento potrebbe condurre ad una soluzione delle aporie teoriche e della difficile utilizzazione pratica che caratterizzano le tipologie di cui abbiamo discusso.

Su questa strada possiamo distinguere due alternative: quella che utilizza come criterio di costruzione della tipologia la distribuzione dei costi e dei benefici e quella, legata più strettamente all'approccio dell'economia del benessere, che si focalizza sulla distinzione tra beni individuali e beni collettivi.

Per quanto concerne la prima alternativa, la proposta classificatoria più nota e meglio articolata è quella avanzata da James $\mathrm{Q}$. Wilson (1973), e dallo stesso autore approfondita a livello di analisi empirica nel contesto delle politiche regolative (Wilson 1974; 1980). Wilson distingue dapprima tra l'adozione di una nuova politica ed il cambiamento di una già esistente. Tralasciamo, ci 


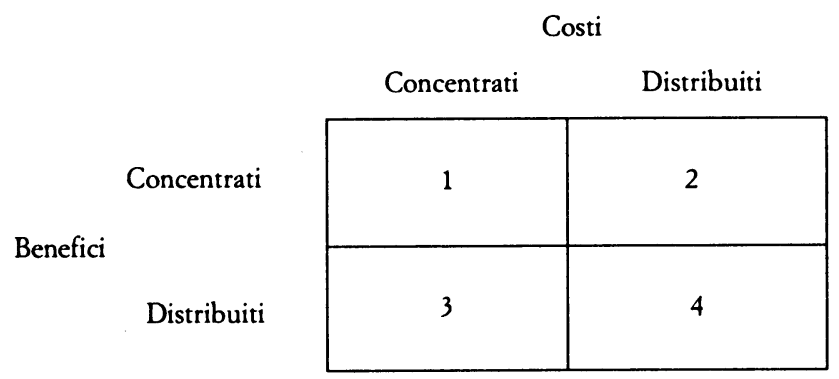

FIG. 2. La tipologia delle politiche di Wilson (1973)

torneremo successivamente, il primo elemento della distinzione e soffermiamoci sui processi decisionali che si caratterizzano per apportare modificazioni a politiche già esistenti: in questo ambito le politiche - o meglio le proposte di policy (Wilson 1974) - possono essere classificate individuando la distribuzione dei costi e dei benefici dal punto di vista dei destinatari delle decisioni. I costi ed i benefici possono essere sia tangibili che intangibili.

I costi ed i benefici vengono dicotomizzati, cioè ridotti a due modalità, eguali per entrambi gli attributi: la concentrazione e la distribuzione (nel senso di diffusione). Combinando queste quattro modalità si ottiene uno spazio di attributi che prevede quattro tipi di politiche (fig. 2).

Il tipo 1 (costi concentrati/benefici concentrati) è caratterizzato per generare un conflitto organizzato e reiterato, in cui i ben delimitati gruppi dei beneficiari e dei deprivati si contrappongono in tutte le fasi del processo decisionale, dalla formulazione all'implementazione. Il tipo 2 (costi distribuiti/benefici concentrati) individua le politiche in cui vengono forniti benefici ad un determinato settore o gruppo di interesse, senza che venga imposto chiaramente un costo ad un altro ben delimitato gruppo di interesse. In questo caso, $\mathrm{i}$ beneficiari sono incentivati ad organizzarsi ed a fare opera di pressione sul sistema politico. $\mathrm{Si}$ deve sottolineare come questo tipo non individui processi unicamente distributivi, ma possa anche identificare politiche che nella terminologia lowiana possono essere ritenute regolative ${ }^{10}$. Il tipo 3 (costi concentrati/benefici distribuiti) comprende quelle

10 Pensiamo, ad esempio, alla determinazione di certi requisiti per poter accedere a determinate cariche pubbliche, oppure a vincoli posti per l'accesso ad un determinato settore produttivo o, ancora, agli albi professionali (La Spina 1989, 301; Stigler 1971). 
politiche che impongono vincoli, ritenuti indispensabili per fornire un beneficio alla collettività, a determinati settori produttivi, associazioni d'interesse, associazioni professionali. Queste politiche possono manifestarsi, secondo Wilson, o in momenti di crisi sistemiche o grazie all'azione di un imprenditore politico capace di stimolare l'opinione pubblica. Gli oppositori hanno, però, il vantaggio di essere incentivati ad organizzarsi, proprio perché pagano visibilmente i costi, e possiedono notevoli possibilità di rendere inefficace la decisione nella fase d'implementazione, catturando l'apparato amministrativo di riferimento, mentre il fronte dei promotori si sfalda, a causa della diffusione dei benefici ${ }^{11}$. Il tipo 4 (costi distribuiti/benefici distribuiti) identifica quelle proposte di policy che si caratterizzano per la prevalenza di grandi organizzazioni - soprattutto, ma non solo, dei partiti politici. Queste politiche possono essere sia assolutamente aconflittuali e del tutto incontroverse (a causa della difficoltà ad individuare un ambito più ristretto di beneficiari), sia decisamente conflittuali, vista la natura degli attori, fortemente organizzati, che partecipano al processo (nel momento in cui sull'issue in questione vi siano riferimenti valoriali differenti) ${ }^{12}$.

Questa proposta tipologica risolve alcune delle ambiguità presenti nelle tipologie fondate sulla coercizione. Essa, soprattutto, sembra soddisfare in modo più adeguato il requisito della mutua esclusività delle classi: distinguendo le politiche in base alla concentrazione/distribuzione dei costi e dei benefici, sembrano potersi superare le difficoltà classificatorie e le tendenze

11 Wilson sottolinea come una politica di questo tipo tenda a manifestarsi raramente nella realtà, ovvero a trasformarsi in fieri in una politica a benefici concentrati e costi diffusi. Ciò, poiché essa implica, da una parte, una forte attenzione dell'opinione pubblica, che deve essere mantenuta costante, dall'altra un incentivo ad organizzarsi per gli interessi che vengono chiamati a pagare i costi della decisione. Nella realtà, tali politiche vengono formulate sull'onda di una mobilitazione di interessi diffusi ed in presenza di un imprenditore politico. Nella fase di attuazione, quando la mobilitazione scema e viene meno l'attenzione dei mass-media e dell'opinione pubblica, gli interessi dapprima perdenti riescono ad imporsi, catturando l'agenzia preposta ad attuare la normativa (Wilson 1973, 334-5). Sul punto si veda anche Pennock (1979).

12 E questo il caso in cui l'acutizzarsi del conflitto e, quindi, l'imporsi di logiche partigiane, deriva dall'emergere di questioni che mettono in gioco l'identificazione ideologica o i valori di organizzazioni e di gruppi, incentivando la ridefinizione ideologica dei costi e dei benefici. I casi più evidenti, ed eclatanti, sono quelli dei diritti civili e sociali, del divorzio, dell'interruzione volontaria della gravidanza, dell'eutanasia. Ricordiamo, però, come dinamiche di tal fatta possano instaurarsi anche in altri settori, ad esempio quello economico (pensiamo, ad esempio, nel caso italiano, alla questione della scala mobile). 
a cadere, in definitiva, nelle catalogazioni formal-istituzionali connesse alle categorie lowiane. Per contro, come è stato giustamente sottolineato, la tipologia avanzata da Wilson ha almeno due problemi di fondo, che incidono notevolmente sulla valutazione e la misurazione dei costi e dei benefici (Gustavsson 1980). Da una parte, non convince la distinzione tra new policies e politiche già esistenti, poiché in molti sistemi democratici occidentali è difficile riscontrare nuove politiche quanto, piuttosto, un diverso modo di trattare le politiche già esistenti; dall'altra, non risulta soddisfacente l'individuazione del policy change come unità di analisi del ricercatore: come trattare, in questo caso, tutte quelle politiche che si sviluppano, inerzialmente, in quanto prodotto di decisioni precedenti ${ }^{13}$ ?

$\mathrm{Al}$ di là di tali elementi problematici, l'eleganza e la precisione analitica della proposta di Wilson ha fatto sì che essa si sia imposta, andando contro alle affermazioni dello stesso auto$\mathrm{re}^{14}$, come la principale alternativa alla proposta tipologica di Lowi offrendo, in effetti, uno schema certamente affascinante e potenzialmente foriero di interessanti risultati analitici. Il criterio dei costi-benefici, secondo l'approccio proposto da Wilson, è diventato uno strumento analitico assai utilizzato dagli studiosi di policy, soprattutto nel settore dell'analisi empirica e teorica delle politiche regolative. Al tempo stesso, il lavoro di Wilson è stato considerato da molti la strada da percorrere per risolvere le problematiche connesse alle tipologie fondate sul criterio del-

13 La soluzione a questi problemi che è stata avanzata dallo stesso Gustavsson - e cioè la riformulazione in termini marginalisti della proposta tipologica di Wilson, laddove la tipologia lowiana è concepita come indicatore strutturale della politica (individuando le relazioni tra cittadini e governo) - in effetti risulta un'estensione logica dei lavori di Wilson sulle politiche regolative $(1974 ; 1980)$. Infatti, dopo aver riformulato le categorie lowiane nei termini di relazioni tra cittadini e governo, egli applica a ciascuna di esse lo schema classificatorio fondato sulle due dimensioni del rapporto costi/benefici. La proposta, nel complesso, risulta abbastanza interessante, mancando, però, di un'adeguata applicazione ai casi empirici (prescindendo dall'analisi del caso svedese operata dallo stesso autore). Pur apprezzandone le possibilità euristiche, lascia piuttosto dubbiosi l'assunto di fondo, ovvero la definizione della policy come un insieme delle relazioni tra cittadini e potere politico strutturalmente determinato, attraverso il quale il governo induce $\mathrm{i}$ cittadini in modo tale che essi non potrebbero fare diversamente (Gustavsson 1980, 136); tale definizione, in effetti, riformula in modo più articolato l'assunzione di Lowi che individua nella coercizione l'elemento caratterizzante delle politiche pubbliche.

14 Wilson si proponeva non tanto di confutare la proposta classificatoria di Lowi, quanto piuttosto, partendo dal medesimo assunto teorico di fondo - che la policy determina la politics - di risolvere i problemi metodologici e le ambiguità che la tipologia di Lowi presenta (Wilson 1973, 328-330). 
la coercizione, anche se, spesso, le proposte avanzate si differenziano dal punto di vista sostenuto da Wilson.

E questo il caso, ad esempio, di quegli autori che utilizzano la distinzione tra beni privati, o individuali, e pubblici, o collettivi, per classificare le politiche pubbliche. Ad esempio, Kjellberg (1977), pur facendo salve le quattro categorie di policies lowiane, riformula la costruzione della tipologia, sostituendo la dimensione relativa all'ambito di conduzione della coercizione con la dimensione connessa ai beni distribuiti che possono essere, appunto, individuali o collettivi. L'altro criterio della tipologia di Lowi, la probabilità della coercizione, viene trasformato (per conformarsi al criterio fondato sulla natura del bene distribuito), più che sostituito nella sostanza, nel tipo di allocazione, che può essere diretta o indiretta. Su queste basi avremo una politica distributiva in presenza di un'allocazione diretta di un bene individuale; una politica regolativa con l'allocazione indiretta di un bene individuale; una politica costitutiva nel caso di un'allocazione indiretta di un bene collettivo e, infine, una politica redistributiva in presenza di un'allocazione diretta di un bene collettivo.

L'operazione di sostituzione aspira a mantenere ferma la natura delle categorie classificatorie dello schema lowiano, esplicitandone però la valenza disaggregativa e, quindi, diminuendo le aporie che ne informano i principi costitutivi, in particolare attraverso l'individuazione di un criterio ritenuto oggettivo - la natura del bene allocato. Di fatto, però, la riproposizione in termini olsoniani della quadripartizione lowiana sembra, piuttosto, un'opera cosmetica che non rimuove i problemi di fondo che abbiamo precedentemente sottolineato - legati alla presenza, seppur implicita, del criterio della coercizione -, ed aggiungendone degli altri, strettamente connessi alla questione dell'oggettiva possibilità di distinguere tra beni individuali e collettivi. Laddove, infatti, si assumesse che la natura dei beni allocati non è intrinseca ai beni stessi, bensì una costruzione sociale (Malkin e Wildavsky 1991), allora è evidente che rientrerebbero in gioco quelle caratteristiche di soggettività, connesse con la percezione degli attori partecipanti, che Kjellberg si preoccupa di eliminare, attraverso l'introduzione nella tipologia lowiana del criterio fondato sulla natura del bene.

Ancor più ancorata, almeno nelle intenzioni, agli assunti della teoria economica sulla natura dei beni, è la proposta di Kellow (1988), che individua quattro tipi di politica (redistribu- 


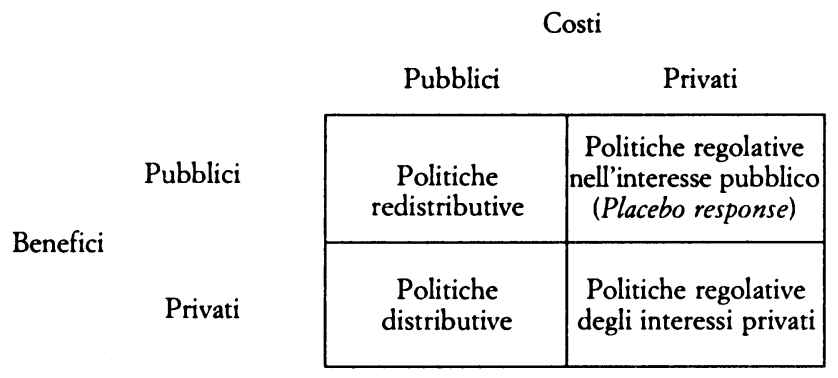

FIG. 3. La tipologia di Kellow (1988)

tivo, distributivo, regolativo degli interessi privati e regolativo nell'interesse pubblico) distinguendo tra costi e benefici in base alla dicotomia pubblico/privato (fig. 3 ).

Questa proposta classificatoria, aspirando anch'essa a mantenere valida la forza euristica delle categorie lowiane, in realtà ne distorce la logica, addivenendo a conclusioni difficilmente condivisibili ${ }^{15}$. Kellow assume, per fondare la propria elaborazione tipologica, che esista omogeneità tra il criterio della percezione dei costi e dei benefici dei destinatari proposto da Wilson, e quello che distingue tra bene, e costi, pubblici e privati; il che è francamente poco condivisibile. Come affermato seccamente dallo stesso Wilson «un beneficio largamente distribuito può essere o può non essere quello che gli economisti definiscono un bene collettivo... Tutti i beni collettivi, come la difesa nazionale, sono un beneficio largamente distribuito, ma non tutti i benefici largamente distribuiti, come i pagamenti della sicurezza sociale, sono beni collettivi» (Wilson 1973, 332). La distinzione tra privati e pubblici dei benefici e dei costi sembrerebbe aumentare l'oggettività dei criteri e, quindi, la delimitazione delle categorie a livello operativo; ciò, poiché, a differenza dello schema wilsoniano - che deve, comunque, individuare le percezioni dei destinatari lasciando, quindi, ampio spazio alla soggettività del ricercatore - in quest'approccio la distinzione

1s Il contributo di Kellow ci sembra insoddisfacente soprattutto nella costruzione della sua proposta tipologica che, pur ispirandosi alla tipologia di Wilson, risulta meno convincente riducendone le potenzialità analitiche; per contro, la sua impostazione relativa ai problemi connessi alla dinamica del processo di policy, con l'enfasi che egli ripone sul problema della scelta delle alternative da parte degli attori, ci pare particolarmente azzeccata e stimolante. 
non è fondata sulla percezione degli attori rispetto alla politica in atto, bensì sulla natura stessa, ritenuta oggettivamente data, dei benefici e dei costi. Ora, anche assumendo come fondata la distinzione tra beni pubblici e privati - in base ai criteri dell'inesclusività, indivisibilità e non-rivalità che caratterizzerebbero i primi rispetto ai secondi (Samuelson 1954; Head 1962; Olson 1965) - la pretesa di voler costruire una tipologia delle politiche incrociando questa distinzione con quella uguale relativa ai costi (anch'essi distinti tra privati e pubblici) risulta creare molte più questioni di quelle che vorrebbe risolvere.

Il tentativo di Kellow, infatti, introduce profondi problemi di ordine metodologico e terminologico. Ad esempio, il tipo redistributivo viene associato a politiche caratterizzate da benefici e costi entrambi pubblici; ciò mentre, sia ragionando nei termini proposti da Lowi (ed anche in quelli di Wilson), sia in base al comune buon senso, le politiche redistributive si caratterizzano proprio per il carattere della forza coercitiva dell'intervento a scapito di determinati settori o classi sociali, in un contesto in cui $\mathrm{i}$ benefici ed i costi tendono ad essere fortemente concentrati, trattandosi di un classico processo a somma-zero, in cui la quantità dei benefici e dei costi è sì molto elevata, ma fortemente divaricata (laddove l'ampiezza dei settori sociali coinvolti non pregiudica ma, anzi, enfatizza la difficoltà a disaggregare la ripartizione dei costi e dei benefici). Certo, accettando la definizione olsoniana, per cui un bene può essere collettivo per un intero gruppo sociale, ma individuale per altri (Olson 1965, trad. it. 1983, 27), il discorso potrebbe anche funzionare. Il problema è che, in realtà, Kellow concepisce il bene pubblico non nell'accezione articolata e selettiva di Olson, bensì nel senso di bene dell'intera collettività nazionale (per cui, specularmente, un costo pubblico è un costo dell'intera collettività). Ad esempio, egli considera le politiche redistributive e quelle distributive come assai simili ed omogenee nel lato dei costi, ritenendo che la fonte delle risorse per entrambe le politiche sia il sistema fiscale generale (Kellow 1989, 540); il che lascia estremamente perplessi, poiché le politiche redistributive tendono a scaricare i costi non tanto sulla collettività quanto, piuttosto, sul settore sociale che risulta perdente in questo tipo di allocazione di valori.

Di poi, la proposta di scindere le politiche regolative in due tipi - quello dell'interesse privato e quello dell'interesse pubblico (definito anche placebo response) - risulta discutibilissima e troppo valutativa, poiché tende ad escludere, per definizione, la 
possibilità che regolazioni nell'interesse pubblico possano essere, se non attuate, almeno formulate ${ }^{16}$. La verità è che a non risultare assolutamente convincente è proprio la scelta e la scarsa pulizia analitica dei criteri adottati per costruire tale classificazione $^{17}$.

\section{Le tipologie fondate su dimensioni sistemiche e strutturali}

I due tipi di classificazione tipologica che abbiamo sin qui affrontato sono caratterizzati, per lo più, dall'individuazione di criteri strettamente connessi con il fenomeno che si vuole classificare: sono, cioè, elementi ritenuti propri del concetto di policy a costituire gli assi portanti per la sua suddivisione in classi. Rispetto a questa caratteristica omogenea, meritano attenzione quei tentativi di costruire classificazioni delle politiche pubbliche che prendono in considerazione dimensioni sistemiche e/o strutturali, esterne al fenomeno che si intende classificare. $\mathrm{Ci}$ riferiamo, in particolare, ai lavori di Salisbury (1968), Salisbury e Heinz (1970) e Hayes (1978). Salisbury, nel suo primo contributo, mantiene ferma la tripartizione originaria di Lowi, enucleando dal tipo regolativo una quarta categoria, quella di politica autoregolativa, e costruendo una classificazione fondata sulla frammentazione/integrazione di due dimensioni sistemiche: la domanda sociale e il sistema decisionale ${ }^{18}$.

All'interno della logica di questa costruzione tipologica vi è notevole ambiguità rispetto ai termini di integrazione e frammentazione; tale caratteristica si riflette direttamente soprattutto

16 In questo senso, la distinzione quadripartita delle politiche regolative formulata da Wilson (1974) risulta certamente di livello e di qualità incomparabilmente superiore. Per una attenta disamina della letteratura e delle problematiche connesse al tipo regolativo si rimanda a Giuliani (1992).

${ }^{17}$ Nella sostanza, a non convincerci affatto sono tutte le tipologie delle politiche pubbliche assolutamente ancorate all'assunto economicistico che presuppone una natura oggettiva dei beni. Tra queste ricordiamo, ad esempio, la tipologia che distingue tra beni privati, comuni, strumentali e pubblici (Ostrom e Ostrom 1978); oppure quella, molto articolata, che distingue otto tipi di policy (quattro regolativi e quattro distributivi), fondata essenzialmente sulla dimensione dell'indivisibilità del bene - dicotomizzata in indivisibilità di produzione ed indivisibilità di consumo - che viene incrociata con le caratteristiche del target di riferimento (che può essere individuale o di gruppo) (Champney 1988).

${ }_{18}$ Il tipo distributivo è determinato da una domanda e da un sistema decisionale entrambi frammentati; quello regolativo da una domanda frammentata e da un sistema decisionale integrato; quello redistributivo da una domanda e da un sistema decisionale entrambi integrati; infine, il tipo auto-regolativo è determinato da una domanda integrata e da un sistema decisionale frammentato (Salisbury 1968, 171). 
su una delle dimensioni, quella del sistema decisionale che, in effetti, sembra risultare determinante per l'individuazione dei tipi di policy. Se si accetta come esempio di sistema integrato quello ad esecutivo forte (o quello di una struttura amministrativa fortemente gerarchica) e, viceversa, come esempio frammentato quello delle assemblee legislative (Salisbury 1968, 168), allora si dovrebbe accettare come conseguenza che le assemblee legislative hanno solo due alternative di policy, quella distributiva e quella autoregolativa, mentre agli esecutivi forti restano le altre due possibilità, quella regolativa e quella redistributiva. Ciò è francamente poco convincente e, in effetti, successivamente lo stesso Salisbury tende a correggere questo aspetto della propria classificazione, sostituendo la dimensione della frammentazione/integrazione del sistema decisionale con quella dei costi che i decisori debbono pagare per produrre la decisione. I costi individuati sono: l'utilità, positiva e negativa, per i decisori, di agire in modo tale da conferire un beneficio a qualche constituency rilevante; i costi informativi, derivati dalla necessità di assumere le conoscenze sufficienti su una questione in modo tale da poter esprimere una posizione; il tempo, l'energia e le risorse necessarie per negoziare una coalizione vincente (Salisbury e Heinz 1970, 42-43). In base a questa dimensione, viene operata una distinzione dicotomica tra le possibili politiche che verranno perseguite: da una parte stanno le politiche allocative, caratterizzate da bassi costi decisionali, che distribuiscono benefici materiali e simbolici ad individui e gruppi; dall'altra parte sono individuate le politiche strutturali, ovvero quelle politiche che, essendo alto il costo decisionale per formare una coalizione vincente, non assegnano direttamente valori ma stabiliscono regole o strutture d'autorità finalizzate a porre in essere, successivamente, politiche allocative. Queste due classi possono essere articolate al loro interno, incrociando la dimensione dei costi con quella della domanda (fig. 4). Le politiche allocative avranno perciò un carattere distributivo in presenza di una domanda frammentata e un carattere redistributivo in presenza di una domanda integrata. Dal canto loro, le politiche strutturali possono essere distinte in regolative ed autoregolative a seconda che sia riscontrabile frammentazione o integrazione nella domanda (Salisbury e Heinz 1970, 48-49).

Lo schema tipologico così modificato risulta, tuttavia, seppur meglio impostato, ancora insoddisfacente ed equivoco in alcuni punti fondamentali. In primo luogo, pur risolvendone 


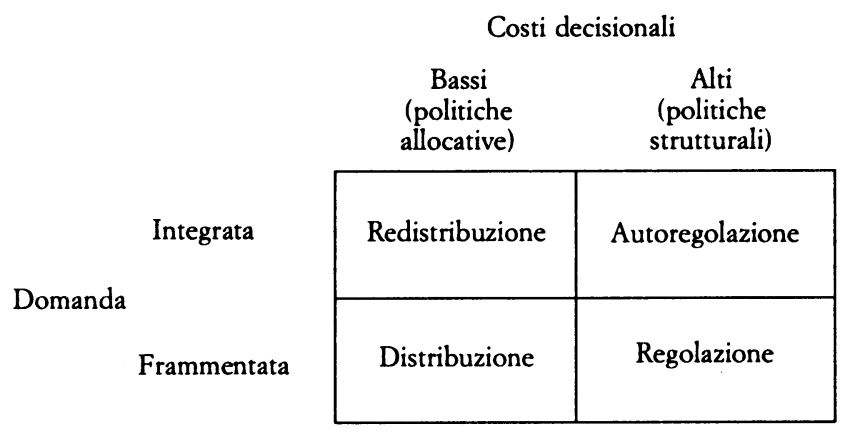

FIG. 4. La tipologia di Salisbury e Heinz (1970)

l'ambiguità riscontrata precedentemente, la scelta di definire l'integrazione della domanda in relazione «all'accordo dei gruppi che richiedono una decisione di policy» (Salisbury e Heinz $1970,48)$, quindi in termini strettamente ed unicamente cooperativi, lascia abbastanza perplessi soprattutto in relazione al tipo redistributivo. Esso, infatti, risulterebbe, in questa impostazione, totalmente deprivato delle potenzialità conflittuali che solitamente ad esso, nella letteratura politologica sia teorica che empirica, vengono imputate (non a caso l'elevata potenzialità di conflitto che caratterizza la natura delle politiche redistributive comporta una loro rara concretizzazione, ovvero un'implementazione molto blanda spesso mitigata da connotazioni distributive).

D'altra parte, la stessa suddivisione delle politiche tra allocative e strutturali, in base al criterio dei costi per i decisori, risulta assai poco convincente, in relazione soprattutto alle politiche regolative. Queste, infatti, non necessariamente non allocano direttamente benefici materiali o simbolici, così come non necessariamente la decisione di delegare agli apparati amministrativi la regolazione del conflitto significa prendere decisioni strutturali, poiché all'interno della decisione primaria può essere chiaramente definita la procedura d'implementazione. Allo stesso modo, considerare le politiche redistributive a basso costo decisionale, in quanto il costo per raggiungere la decisione viene considerato inferiore al volume di valori scambiati (Salisbury e Heinz 1970, 48), risulta un'operazione di drastica, e forse eccessiva, riduzione della complessità intrinsecamente connaturata a tali processi decisionali. Evidentemente, anche la 
Domanda

\begin{tabular}{|c|c|c|c|}
\hline \multirow{2}{*}{\multicolumn{2}{|c|}{ Nessun provvedimento }} & $\begin{array}{l}\text { Consensuale } \\
\text { (a somma } \\
\text { positiva) }\end{array}$ & $\begin{array}{l}\text { Conflittuale } \\
\text { (a somma } \\
\text { zero) }\end{array}$ \\
\hline & & $\begin{array}{l}\text { Non interferenza } \\
\text { (laissez-faire) }\end{array}$ & $\begin{array}{c}\text { Non decisione } \\
\text { (soppressione del conflitto) }\end{array}$ \\
\hline \multirow[t]{2}{*}{ Offerta } & $\begin{array}{c}\text { Delega } \\
\text { (Policy without law) }\end{array}$ & $\begin{array}{c}\text { Autoregolazione } \\
\text { (autonomia legittimata) }\end{array}$ & $\begin{array}{c}\text { Regolazione } \\
\text { (estensione del conflitto) }\end{array}$ \\
\hline & $\begin{array}{c}\text { Decisione } \\
\text { (rule of law) }\end{array}$ & $\begin{array}{c}\text { Distribuzione } \\
\text { (pork barrel politics) }\end{array}$ & $\begin{array}{c}\text { Redistribuzione } \\
\text { (trasferimento di risorse) }\end{array}$ \\
\hline
\end{tabular}

Fig. 5. La tipologia di Hayes (1978)

definizione del secondo criterio, quello dei costi decisionali, risulta particolarmente poco appropriata, soprattutto perché non tiene conto sufficientemente sia dei costi/benefici per i destinatari della politica, sia delle condizioni strutturali e contingenti che possono costituire vincoli o risorse per ottenere una coalizione vincente.

Nella sostanza, quindi, i criteri scelti per costruire questa tipologia risultano quantomeno poco convincenti, individuando tipi di politiche pubbliche che, pur mantenendo etichette eguali a quelle proposte da altre classificazioni, ne modificano il contenuto in modo discutibile e assai poco adeguato al riscontro empirico. In realtà, la tipologia di Salisbury e Heinz tende a privilegiare, seppur implicitamente, la dimensione della domanda (dicotomizzata in modo decisamente insoddisfacente), e ha ragione, allora, chi sottolinea come il criterio dei costi per i decisori ci possa aiutare assai poco per specificare le caratteristiche di un processo decisionale (Uslaner 1985).

Un tentativo di risolvere, rielaborandole, i problemi connessi alle tipologie appena discusse, è quello avanzato da Hayes. Nel suo schema tipologico, fondato sull'assunto di un ruolo determinante dei gruppi di pressione, egli individua sei processi di produzione delle politiche pubbliche (fig. 5), mantenendo la dimensione della domanda - distinta però tra consensuale $\mathrm{e}$ conflittuale - ed introducendo la dimensione dell'offerta legislativa, che viene suddivisa in tre modalità: la non-decisione (no 
bill), la decisione ambigua che tende a delegare alla fase di implementazione la scelta definitiva (policy without law) e la decisione che alloca esplicitamente risorse sia materiali che valoriali (rule of law).

Risulta subito chiaro come, anche in questa proposta tipologica, la dimensione chiave sia quella della domanda. Avremo, infatti: una politica di non interferenza se i gruppi di interesse sono d'accordo per impedire qualsivoglia ingerenza governativa; una politica autoregolativa quando un gruppo, o una coalizione di gruppi cerca di ottenere il riconoscimento legale della propria autonomia; infine, avremo una politica distributiva quando un gruppo ricerca l'allocazione di un beneficio e, in assenza di un'opposizione attenta, lo ottiene. Dall'altra parte, in presenza di una domanda conflittuale si riscontrerà: una non decisione quando gruppi ed élites assai munite riescono ad influenzare l'opinione pubblica e le istituzioni politiche in modo da limitare ai minimi termini l'agenda; una politica regolativa, discrezionale e simbolica, quando la strategia della non decisione viene aggirata da gruppi abbastanza forti da imporre, comunque, una decisione agli organi istituzionali; una politica redistributiva quando, a differenza di quanto accade nell'arena distributiva, il gioco non può essere condotto a somma positiva, poiché anche i potenziali perdenti sono attenti allo svolgimento del processo decisionale.

E particolarmente interessante sottolineare le caratteristiche imputate da Hayes al tipo distributivo ed a quello regolativo. Le politiche distributive non si caratterizzano per la disaggregabilità quasi infinita dei benefici (dovuta al meccanismo delle mutue non-interferenze) quanto, piuttosto, per il fatto di poter escludere alcuni potenziali giocatori così che «la torta dei benefici può essere allargata alle spese di questi attori inattivi nel caso sia necessario accomodare un potenziale opponente» (Hayes 1978, 147). Le politiche regolative anche in questo caso, come nella tipologia precedente, sono caratterizzate da un'intrinseca natura simbolica, e dalla tendenza a spostare il conflitto in ambito amministrativo o giurisdizionale.

Sono, perciò, le caratteristiche della domanda che determinano il fatto che una decisione non venga o venga presa; e, nel secondo caso, sono sempre le caratteristiche della domanda che determinano l'esito del processo decisionale e la formalizzazione dell'output legislativo. È la domanda che determina l'offerta in modo quasi automatico, in un contesto teorico che presuppone un rapporto di quasi completa dipendenza degli attori po- 
litico-istituzionali rispetto ai gruppi di interesse e di pressione. In verità, l'esaltazione del ruolo della domanda, che nasce dall'assunto di fondo su cui giace tale tipologia (i gruppi di interesse «contano», non sono le caratteristiche della policy a determinare la politica), riduce ad un ruolo accessorio il secondo criterio, le caratteristiche della decisione formalizzata. Pochissima attenzione viene dedicata alle caratteristiche e alle risorse dei decisori pubblici, reificati nella fissità delle categorie dell'output, laddove, pur ipotizzando che gli attori politici possano controbilanciare il peso e le pressioni dei gruppi organizzati a vantaggio di quelli non organizzati (Hayes 1978, 161; ma anche Schattschneider 1975, 48 ss.), questa possibilità viene ridotta a pura residualità ed eccezionalità, all'interno di una classificazione tipologica vigorosamente ancorata alla dimensione della domanda.

La caratteristica delle tipologie fondate su dimensioni sistemiche e strutturali consiste, soprattutto, nel considerare il processo di policy come assolutamente, ed oggettivamente, determinato da fattori sistemici, soprattutto dalla domanda sociale ${ }^{19}$, eliminando qualsiasi possibilità di autonomia da parte dei decisori e, quindi, presupponendo una prestrutturazione definitiva del processo decisionale da parte di fattori esterni ad esso. In effetti, ci troviamo in una posizione concettualmente antitetica rispetto a quella di Lowi, per il quale la domanda sociale conta pressoché nulla, mentre risalta l'autonomia dei decisori; l'univocità antitetica di entrambe le impostazioni tende ad attribuire, in modo omogeneo, fissità e staticità alle politiche pubbliche, cristallizzandone il contenuto da una parte su variabili esterne dall'altra su variabili interne.

\section{L'ineludibile staticità delle categorie delle politiche pubbliche}

Abbiamo sin qui delineato criticamente i principali sforzi tipologici prodotti dagli studiosi delle politiche pubbliche al fine

19 Il ruolo centrale della domanda di politiche è affermato anche dagli approcci razionali alla teoria contrattuale. In quest'ottica, sono le difficoltà dei cittadini a risolvere problemi privati come il coordinamento, la divisione e la defezione a rendere necessario l'intervento del governo e, quindi, la produzione delle politiche pubbliche È interessante sottolineare come, su queste basi, la tipologia di Lowi possa essere mantenuta nelle sue caratteristiche categoriali, pur ribaltandone gli assunti di fondo. Sul punto si rimanda al saggio di Heckathorn e Maser (1990). 
di classificare l'oggetto fondamentale della loro indagine teorico-empirica.

Il quadro complessivo che ne deriva risulta particolarmente problematico. La tradizione tipologica che caratterizza l'analisi delle politiche pubbliche offre uno spettro assai variegato e, molto spesso, non-cumulativo di proposte classificatorie. Il campo è caratterizzato, in primis, da una fondamentale rivalità tra le diverse tipologie che tendono a presentarsi come sostanzialmente incompatibili l'una con l'altra (seppure, in alcuni casi, apparentemente simili per quanto concerne la denominazione dei tipi). I criteri scelti per costruire gli schemi classificatori, cioè, costituiscono una discriminante essenziale che conduce, presupponendola, ad una differente prospettiva analitica. Le proposte, come abbiamo cercato di evidenziare, portano ad esiti abbastanza contrastanti, poiché la diversa prospettiva teorica e classificatoria perseguita conduce, spesso, a definizioni qualitativamente differenti dei medesimi tipi di politica pubblica. Il che è certamente un esito poco soddisfacente per un campo d'analisi che abbisogna disperatamente di affinare i propri strumenti analitici e di trovare delle definizioni sufficientemente condivise dai propri cultori.

Nel complesso, poi, la tradizione tipologica presenta una serie di aporie e di problemi concettuali che sussistono con forza, a prescindere dai criteri che vengono scelti e dai «tipi» di tipi che vengono individuati e definiti. Soprattutto due sono i problemi ancora irrisolti, e probabilmente irrisolvibili, delle tipologie delle politiche pubbliche: la tendenziale difficoltà a rispettare la mutua esclusività delle classi e, soprattutto, la staticità nella quale le classificazioni tipologiche imprigionano il fenomeno oggetto di analisi. La tendenza alla staticità delle tipologie delle politiche pubbliche deriva, peraltro, direttamente dal tentativo di rispettare il criterio della mutua esclusività. Proprio per rendere le categorie in cui vengono suddivise le classificazioni mutualmente esclusive, gli studiosi hanno la tendenza a restringere di fatto il significato attribuibile al concetto di policy. In questo senso, risulta assolutamente emblematico il lavoro dell'ultimo Lowi, il quale riduce la politica pubblica semplicemente all'espressione formalizzata della decisione. Questa estrema riduzione costituisce, è bene sottolinearlo, semplicemente il più fulgido manifestarsi di un fenomeno di riduzione definitoria e concettuale che caratterizza la tradizione tipologica delle politiche pubbliche. $\mathrm{Ci}$ riferia- 
mo al fatto, cioè, che ad essere classificata, nella realtà, non è la politica pubblica, pur nei diversi significati che a tale concetto sono stati attribuiti, bensì un determinato elemento della policy: la decisione formalizzata. Tale, probabilmente inevitabile, riduzione comporta una cristallizzazione che estremizza la naturale staticità propria dello strumento tipologico, imprigionando le politiche pubbliche in una fissità che rischia di fuorviare l'analisi di un fenomeno costitutivamente articolato e complesso. Quest'opera, spesso inconsapevole ovvero non esplicitata (tranne che nel caso di Lowi e di Wilson), di riduzione del significato del concetto di policy può essere valutata secondo due punti di vista. Partendo, cioè, dal presupposto che per politica pubblica si debba intendere un fenomeno assolutamente irriducibile ad una decisione discreta, bensì un insieme di attori, strategie, comportamenti, regole e routines, caratterizzato dal fatto di costituire un fenomeno «più esteso» di una singola decisione e di possedere un'intrinseca intenzionalità ${ }^{20}$, la riduzione operata dalla tradizione tipologica origina due riflessioni differenti, pur se non necessariamente antitetiche.

Da una parte, cioè, l'impressione è che sia gli autori delle principali tipologie sia, in misura forse maggiore, i loro critici, abbiano perseguito un'eccessiva, e per certi versi dannosa, ade-

${ }^{20}$ Sul concetto di policy, ovvero il concetto che dovrebbe consentire di definire ed individuare l'oggetto dell'analisi delle politiche pubbliche, non vi è certamente accordo tra gli studiosi. Nella letteratura specialistica abbondano le definizioni, spesso nọ confrontabili e spesso decisamente differenti. In generale, però, ci pare abbia ragione Heclo (1972) nel ritenere che due elementi possano accomunare le più differenti definizioni del concetto di policy: il termine policy caratterizza un fenomeno che è al tempo stesso più esteso di una singola decisione, di un singolo atto, ed è informato da una dichiarata ed esplicita intenzionalità. Questa concordanza su due elementi, comunque significativi, non riesce, ad ogni modo, a creare una adeguata uniformità di definizioni. Soprattutto, sembra esservi disaccordo sul ruolo del potere pubblico, per alcuni attore centrale di una policy (oltre il più volte citato Lowi, si veda Dye 1972) cui va imputata l'intenzionalità del processo decisionale; per altri, invece, esso è solo uno degli attori che, con altri, partecipa al decorso delle politiche laddove, quindi, sono presenti più intenzionalità (Dunn 1981). Inoltre, notevoli divergenze sono riscontrabili sull'estensione del concetto: vi è chi ne privilegia l'aspetto progettuale; chi, invece, ne estende il significato in modo tale da coprire il processo decisionale fino alla produzione dell'output legislativo; chi, in ultimo, include anche l'implementazione (come fa pure chi scrive). Ciò detto, merita sottolineare, comunque, come la forza euristica del concetto di policy possa esplicarsi compiutamente solo se esso è concepito come uno strumento capace di catturare un fenomeno processuale, non una decisione discreta, né, tantomeno, un'intenzionalità programmatica, seppure ben articolata e complessa. 
renza al rigore imposto dalla logica classificatoria. Non si è considerato, quindi, che la natura stessa delle classificazioni tipologiche - il fatto cioè che necessariamente esse implichino una notevole perdita di informazioni rispetto al fenomeno in oggetto - costituisce un ostacolo assai difficile da aggirare in presenza di un fenomeno assolutamente non auto-esplicativo come quello delle politiche pubbliche, ovvero di fronte ad un concetto analitico, quindi sostanzialmente non reificabile, come quello di policy. In questa prospettiva, quindi, l'eccesso di criticismo imputabile forse anche a chi scrive -, magari fondato su argomentazioni più che condivisibili $\mathrm{da}$ un punto di vista rigorosamente metodologico, rischia di oscurare il contributo di chiarificazione concettuale e di supporto linguistico che la tradizione tipologica ha, comunque, offerto.

In effetti, alcuni contributi, penso soprattutto al primo Lowi e a Wilson, seppur insoddisfacenti in un'ottica algidamente ancorata agli assunti della logica classificatoria e del procedimento scientifico rigidamente inteso, hanno costituito pietre miliari per lo sviluppo teorico dei policy studies, individuando concetti, definizioni e strumenti analitici che sono patrimonio comune degli studiosi. È vero, cioè, che essi hanno, in realtà, costruito delle tipologie delle decisioni normative. $\grave{E}$ anche vero, però, che i tipi lowiani e l'applicazione del criterio dei costi benefici utilizzata da Wilson costituiscono degli strumenti analitici impiegati normalmente negli studi di policy, anche estrapolati dal loro modello originario e prescindendo dalla definizione di policy adottata.

D'altra parte, e siamo alla seconda considerazione che ci preme avanzare, la tendenza alla riduzione propria della tradizione tipologica deriva anche dalla pretesa di riuscire a limitare l'ambiguità intrinseca e costitutiva che caratterizza la fenomenologia delle politiche pubbliche. Il fatto, cioè, che non solo possano essere presenti in ogni singola policy, nella medesima fase processuale, elementi riconducibili a diversi tipi, ma anche che la politica stessa possa modificare la propria natura dalla fase di formulazione a quella di implementazione, ovvero che gli esiti della stessa siano assolutamente differenti dagli obiettivi individuati e fissati nella decisione normativa. Perché una politica che è sempre stata regolativa si trasforma in una politica distributiva? Come conciliare il fatto che ad una decisione redistributiva corrisponda un'implementazione fondata su strumenti regolativi? Come spiegare l'assoluta assenza di effetti reali o materiali 
di una decisione di policy ${ }^{21}$ ? Di fronte all'intrinseca ambiguità fenomenologica delle politiche pubbliche, lo strumento classificatorio rischia, se utilizzato in modo dogmatico e poco flessibile, di eliminare uno degli aspetti più interessanti - sia dal punto di vista teorico che analitico - della concreta evoluzione delle politiche pubbliche (Steinberger 1980; Regonini 1985), oscurando quello che dovrebbe essere uno degli obiettivi precipui della ricerca politico-sociale: addivenire a spiegazioni causali.

Inoltre, le classificazioni delle politiche non tentano solo di eliminare l'ambiguità connessa alla concreta dinamica delle politiche ma, ed è questo probabilmente il loro effetto maggiormente discutibile, a causa della staticità a cui riducono la politica pubblica (che già a livello di definizione concettuale viene ridotta ad un fenomeno discreto), unita ad un eccesso di furore esplicativo che non è adeguato a tale strumento metodologico, tendono ad oscurare l'ambito relazionale dal quale la politica viene originata, processata e, nel caso, modificata. Da questo punto di vista la tradizione tipologica intorno alle politiche pubbliche soffre un'impasse teorica che non è risolvibile e nemmeno aggirabile mediante escamotages.

La verità è che lo strumento tipologico per la sua intrinseca natura non deve essere sovrastimato. Esso è indispensabile per una prima ricognizione della realtà, laddove la necessità di impostare il primo approccio analitico rende imprescindibile la perdita di informazioni. Quando, però, ci troviamo davanti ad un corpus decisamente consolidato di conoscenze e di ricerche, lo strumento classificatorio può risultare poco utile ed appropriato allo sviluppo di diverse strategie di ricerca teorica ed empirica ${ }^{22}$. Il problema è quello di capire che nella ricerca scientifica non esistono strumenti buoni o cattivi, bensì strumenti adeguati o non adeguati a quelli che sono i fini dell'indagine. In

${ }^{21}$ La mancanza di effetti reali (rispetto agli obiettivi statuiti) delle politiche può essere imputata - oltre che alle dinamiche conflittuali che possono emergere tra gli attori nella fase di attuazione - o ad un errore di valutazione della tecnologia sociale disponibile da parte dei decisori, oppure ad una scelta, scientemente operata da questi, di decisioni che non si intende attuare; a questo proposito Gustafsson (1983) distingue tra pseudo-politiche e politiche simboliche. Più in generale, sulle politiche simboliche si rimanda ai classici Goodin (1977) e Edelman (1964).

22 Sul punto, risulta assai interessante la tradizione tipologica sulla democrazia. Dopo una serie di notevoli sforzi classificatori fondati su un numero ridotto di dimensioni, è maturata l'esigenza di andare oltre, costruendo modelli più articolati e complessi; si pensi, ad esempio, ai modelli polari di Lijphart (1984). Sul punto si vedano le accurate considerazioni di Morlino (1986, 93-94). 
questo senso, l'errore della tradizione tipologica è stato, soprattutto, quello di enfatizzare la valenza esplicativa ed ipotetica delle classificazioni, oltre a quello di non problematizzare analiticamente la riduzione operata del concetto, e del fenomeno, politica pubblica.

Sia chiaro, non riteniamo assolutamente che le incongruenze e le aporie teorico-empiriche delle principali tipologie delle politiche pubbliche possano autorizzare il ritorno a classificazioni di tipo «nominalistico» come da alcuni auspicato (Freeman 1985, 484). Comunque sia, le classificazioni tipologiche fondate su un processo di astrazione dal dato reale sono uno strumento ed un passaggio logico-analitico fondamentale per impostare e condurre un discorso scientifico sul fenomeno politico. Il fatto è che di fronte all'ambiguità costitutiva del fenomeno politica pubblica, che per alcuni è causa di imprecisione definitoria irrimediabile (Rose 1984, 4-5), l'apparato concettuale ed analitico deve essere particolarmente articolato e difficilmente può adeguarsi ad eccessive semplificazioni. È evidente, peraltro, che l'ambiguità da molti imputata al concetto deriva proprio dall'aspirazione teorica a racchiudere all'interno di un singolo termine un fenomeno composto e complesso: un insieme di azioni compiute da un insieme di attori, finalizzate in qualche modo a rispondere ad un problema collettivo. Un insieme, quindi, di comportamenti, soggetti, fatti, vincoli ed opportunità (collocati necessariamente in una dimensione diacronica) che per essere colto nella sua specificità abbisogna di essere considerato in una prospettiva capace di sottolinearne la dinamica. Di fronte a tali caratteristiche, l'inevitabile staticità propria delle categorie classificatorie focalizza solo un'unica faccia del poliedrico fenomeno, eliminandone quella dinamicità e quella diacronicità intrinseca che lo informano.

Queste nostre riflessioni sono basate sul presupposto che le classificazioni tipologiche delle politiche pubbliche siano necessariamente correlate ai requisiti richiesti dalla logica classificatoria positivista. Se, per contro, si intendessero i tipi di politica, forzando le intenzioni dei proponenti, come idealtipi, molte delle critiche concernenti, soprattutto, la mutua esclusività - ma in parte anche la staticità - delle tipologie delle politiche verrebbero meno. $\mathrm{Ci}$ si porrebbe, però, in una prospettiva analitica e teorica sostanzialmente differente da quella perseguita dai proponenti le tradizionali tipologie che abbiamo discusso, uscendo dal filone tradizionale della logica classificatoria e con- 
cependo, quindi, i tipi di politica pubblica come tipi ideali, o modelli (Boudon 1984; Panebianco 1989), ovvero come quadri concettuali che non debbono contenere la realtà, oppure parti di essa, bensì orientare il ricercatore nella selezione degli elementi rilevanti per spiegare contestualmente il fenomeno oggetto di indagine (Weber 1922b).

D'altra parte, poi, quello idealtipico sembra essere l'uso precipuo che gli studiosi delle politiche pubbliche fanno delle principali tipologie quando devono studiare, a fini descrittivi e/o esplicativi, specifici referenti empirici; nella realtà della ricerca, quindi, i problemi connessi con le lacune logiche delle tipologie tendono ad essere travalicati attraverso un uso diverso delle categorie tipologiche. Questa soluzione, sedimentatasi nella prassi della ricerca, certo nulla toglie al dibattito sulla congruenza logico-classificatoria delle tipologie, delineando comunque strade diverse che possono essere perseguite per utilizzare le imperfette conoscenze teoriche sin qui acquisite nella ricerca su un fenomeno intrigante e complesso come quello delle politiche pubbliche.

Se accettiamo, cioè, l'assunto che l'approccio tipologico alle politiche pubbliche possa essere maggiormente fruttifero concependo i tipi come ideali, allora le tipologie a nostra disposizione possono essere meglio utilizzate per spiegare il decorso, anche in chiave comparata, delle politiche pubbliche. In questo senso, si potrebbe perseguire in modo convinto tale strada, adoperando i contributi dell'approccio tipologico per delineare un numero adeguato di caratteristiche imputabili al medesimo tipo. $\mathrm{Si}$ potrebbe, cioè, operare una contaminatio tra diverse classificazioni tipologiche per modellare una pluralità di elementi idealtipicamente riferibili ad ogni singola politica pubblica ${ }^{23}$. Fra l'altro, tale operazione viene, spesso, attuata, magari non esplicitamente, dagli studiosi nelle loro ricerche empiriche ${ }^{24}$. Reste-

${ }^{23}$ Potremmo, ad esempio, caratterizzare i tipi-ideali in base alle caratteristiche relative: al grado di disaggregabilità, all'ambito sociale di applicazione, alla distribuzione/ concentrazione dei benefici, agli attori coinvolti nelle diverse fasi processuali, al contenuto della legislazione formale, al livello di consensualità/conflittualità della domanda, al sistema decisionale, ecc. Si potrebbero, insomma, creare dei modelli ideali multidimensionali delle politiche pubbliche, utilizzando, e rielaborando, i contributi della tradizione tipologica positivista, eliminando, altresì, gran parte dei problemi metodologici e di sostanza ad essa connessi.

${ }^{24}$ Ad esempio, chi scrive ha, pur senza trarne le estreme conseguenze teorico-analitiche, operato in questo senso, ragionando intorno all'adeguatezza delle principali tipologie per delineare le caratteristiche delle riforme amministrative in Italia (Capano 1992, 331-338). 
rebbe ferma, comunque, la difficoltà connessa alla staticità da cui anche tali modelli pluridimensionali non potrebbero uscire, pur ridimensionandola e rendendola meno vincolante, essendo quella una caratteristica costitutiva dello strumento tipologico.

Il vero problema, su cui in fondo si gioca tutta la questione di cui stiamo trattando, ruota, ovviamente, intorno al concetto di politica pubblica. In particolare, al di là della questione definitoria (in cui l'unica vera differenza tra le definizioni riguarda il ruolo attribuito al governo), il nodo gordiano di tutta la vicenda teorica si situa intorno al problema dell'oggettività del complesso fenomeno che viene definito politica pubblica. Per oggettività della politica pubblica intendiamo la presenza, e la possibilità di individuarle fattualmente, di determinate caratteristiche di contenuto che consentano di definire con precisione il tipo di policy. In sostanza, la prospettiva oggettivistica è quella che reifica totalmente le categorie tipologiche; quella prospetti$\mathrm{va}$, cioè, secondo la quale date le caratteristiche proprie di una politica regolativa, questa non potrà che svilupparsi conformemente alla teoria, essendo processata nella propria arena, secondo determinate modalità e regolarità. Secondo questo ragionamento esistono politiche «oggettivamente» distributive, o regolative, o redistributive ecc...; in una visione ancora più decisa, spesso di inconsapevole eccesso prescrittivo, si imputa questo tipo di oggettività anche alle singole issues, per cui ci sono questioni intrinsecamente distributive o regolative ovvero redistributive e così via.

È evidente che tale oggettività è frutto meramente di ansia normativa oppure prescrittiva. Si può anche assumere che una determinata questione - ad esempio l'esigenza di un intervento ambientale - debba, razionalmente, essere processata nell'arena regolativa secondo le modalità proprie di una politica regolativa (poiché questo deduciamo dal modello teorico di riferimento); se, poi, tale politica non resta nell'ambito imputatogli, e sconfina in un'altra arena - distributiva o simbolica - o nella fase di formulazione oppure in quella di implementazione, non possiamo probabilmente riferire tale sconfinamento alle lacune delle categorie tipologiche. Certo, esse sono lacunose, ma non ha senso addebitare loro la mancata adesione della fenomenologia della politica al quadro teorico di riferimento. Se una politica, che in linea teorica dovrebbe avere una tendenza processuale redistributiva, si trasforma in fieri in una politica distributiva, riteniamo che ad essere messa in discussione dovrebbe essere la 
presunta oggettività imputata alla politica stessa. Ed in effetti, la strada da perseguire, e che è ormai comune ai policy scholars, è proprio quella di attribuire, in quanto elemento costitutivo ed imprescindibile, una profonda soggettività al fenomeno politica pubblica (cioè a dire che sono i diversi attori che cercano di assegnare il proprio significato alla policy); tale soggettività deve essere catturata e valutata nelle sue incidenze concrete dal lavoro degli studiosi, attraverso strumenti adeguati.

La difficoltà a riscontrare, nella realtà, concretizzazioni dei tipi «puri» di politica dovrebbe condurre - piuttosto che, come abbiamo visto, a creare caselle intermedie capaci di contenere tipi misti - a rivedere la strategia di analisi e di ricerca sulle politiche pubbliche, accettando i limiti, ed anche i benefici, delle classificazioni tipologiche, soprattutto se utilizzate come strumenti idealtipici. Si tratta insomma, se l'obiettivo dell'indagine scientifica è quello di capire e spiegare, di non fossilizzarsi sulla ricerca disperata di classificazioni ulteriormente raffinate ma, piuttosto, di spostare l'ottica analitica dalla statica alla dinamica delle politiche pubbliche (Heclo 1972; Gustavsson 1980; Regonini 1985; Giuliani 1992). Ciò non significa, sia chiaro, abbandonare completamente le conoscenze e le informazioni disponibili grazie alla tradizione tipologica ovvero considerare tale strumento metodologico come irrilevante; piuttosto si tratta, prendendo atto dei limiti propri di qualsiasi tipologia - sia fondata su categorie mutualmente esclusive oppure su tipi ideali di accettare il fatto che tale strumento ben poco ci può dire dell'ambiguità, della soggettività e della dinamicità del processo di policy: esso ci fornisce una serie di fotografie, di stati, di situazioni date - concrete o ideali. Le classificazioni tipologiche sono uno strumento importante per affrontare l'analisi del fenomeno politica pubblica, aiutandoci a delimitarne alcune caratteristiche, non costituendo, però, l'elemento determinate a spiegarlo. La piena comprensione e spiegazione del fenomeno può essere impostata solo attraverso la formulazione di schemi euristici e di teorie capaci di coglierne quegli aspetti costitutivi - dinamicità, soggettività ed ambiguità - che sono appena sfiorati dalla tradizione tipologica. E proprio l'elaborazione teorica e l'approfondimento empirico di simili teorie e schemi euristici costituiscono il fulcro dell'impegno dei policy scholars nell'ultimo decennio. 
Riferimenti bibliografici

Bauer, R.A. et al. (1963), American Business and Public Policy: The Politics of Foreign Trade, New York, Atherthorn Press.

Berelson, B.R., P.F. Lazarsfeld e W.N. McPhee (1951), Voting, Chicago, University of Chicago Press.

Boudon, R. (1984), La place du désordre, Paris, Presses Universitaires de France; trad. it. Il posto del disordine, Bologna, Il Mulino, 1985.

Bressers, H. e M. Honigh (1986), A Comparative Approach to the Explanation of Policy Effects, in «International Social Sciences Journal», 108, pp. 267-287.

Calise, M. (1987), Organizzare le politiche: arene di partito ed arene amministrative a Napoli, in «Democrazia e diritto», n. 6, pp. 163-187.

Capano, G. (1992), L'improbabile riforma. Le politiche di riforma amministrativa nell'Italia repubblicana, Bologna, Il Mulino-Arel.

Champney, L. (1988), Public Goods and Policy Types, in «Public Administration Review», v. 48, pp. 988-994.

Dente, B. (1990), Le politiche pubbliche in Italia, in B. Dente (a cura di), Le politiche pubbliche in Italia, Bologna, Il Mulino, pp. 9-47.

Dunn, W.N. (1981), Public Policy Analysis: An Introduction, Englewood Cliffs, Prentice Hall.

Dye, T.R. (1972), Understanding Public Policy, Englewood Cliffs, Prentice Hall.

Edelman, M. (1964), The Symbolic Uses of Politics, Chicago, University of Illinois Press; trad. it. Gli usi simbolici della politica, Napoli, Guida, 1987.

Eulau, H. e R. Eyestone (1968), Policy Maps of City Councils and Policy Outcomes: A Developmental Analysis, in «American Political Science Review», LXII, pp. 126-143.

Ferrera, M. (1989), Le politiche pubbliche, in L. Morlino (a cura di), Scienza politica, Torino, Edizioni della Fondazione Agnelli, pp. 241-256.

Freeman, G.P. (1985), National Styles and Policy Sectors: Explaining Structured Variation, in «Journal of Public Policy», V, pp. 467496.

Froman, L.A. (1967), An Analysis of Public Policies in Cities, in «Journal of Politics», XXIX, pp. 94-108.

- (1968), The Categorization of Policy Content, in A. Ranney (a cura di), Political Science and Public Policy, Chicago, Markham, pp. 4152.

Giuliani, M. (1992), Politiche regolative. Fra scambi e giochi, in «Rivista italiana di Scienza politica», XXII, pp. 335-374.

Goodin, R. (1977), Symbolic Rewards: Being Bought Off Cheaply, in «Political Studies», XXV, pp. 383-396. 
Greenberg, G.D. et al. (1977), Developing Public Policy Theory, Perspectives from Empirical Research, in «American Political Science Review», LXXXI, pp. 1532-1543.

Gustafsson, G. (1983), Symbolic and Pseudo Policies as Responses to Diffusion of Power, in «Policy Sciences», XV, pp. 269-287.

Gustavsson, S. (1980), Types of Policy and Types of Politics, in «Scandinavian Political Studies», III, pp. 123-142.

Hart, H.L.A. (1961), The Concept of Law, New York, Oxford University Press; trad. it. Il concetto di diritto, Torino, Einaudi, 1965.

Hayes, T.H. (1978), The Semi-Sovereign Pressure Groups: A Critique of Current Theory and an Alternative Typology, in «The Journal of Politics», XL, pp. 134-161.

Head, J.G. (1962), Public Goods and Public Policy, in «Public Finance», XVII, pp. 197-219.

Heckathorn, D.D. e S.M. Maser (1990), The Contractual Architecture of Public Policy: A Critical Reconstruction of Lowi's Typology, in «Journal of Politics», LXII, pp. 1101-1123.

Heclo, H. (1972), Review Article: Policy Analysis, in «British Journal of Political Science», II, pp. 83-108.

Hill, K.Q. e J.P. Plumlee (1984), Policy Arenas and Budgetary Politics, in «Western Political Quarterly», XXXVII, pp. 84-99.

Hofferbert, R. (1974), The Study of Public Policy, New York, Bobbs Merrill.

Huntington, S.P. (1961), The Common Defense, New York, Columbia University Press.

Kellow, A. (1988), Promoting Elegance in Policy Theory: Simplifying Lowi's Arenas of Power, in «Policy Studies Journal», XVI, pp. 713-724.

- (1989), Taking The Long Way Home? A Reply to Spitzer on the Arenas of Power, in «Policy Studies Journal», XVII, pp. 537-549.

Kjellberg, F. (1977), Do Policies (Really) Determine Politics? And Eventually How?, in «Policy Studies Journal», V, pp. 554-570.

La Spina, A. (1989), La decisione legislativa, Milano, Giuffrè.

Lijphart, A. (1984), Democracies, London, Yale University Press; trad. it. Le democrazie contemporanee, Bologna, Il Mulino, 1988.

Lowi, T.J. (1964a), American Business, Public Policy, Case Studies, and Political Theory, in «World Politics», XVI, pp. 677-715.

- (1964b), At the Pleasure of the Mayor, New York, Free Press.

- (1966), Distribution, Regulation, Redistribution: The Function of Government, in R.B. Ripley (a cura di), Public Policies and their Politics, New York, Norton Co.

- (1967), Making Democracy Safe for the World, in J. Rosenau (a cura di), Domestic Sources of Foreign Policy, New York, Free Press, pp. 295-331.

- (1969), The End of Liberalism, New York, Norton Co. 
- (1970), Decision Making vs. Policy Making, in «Public Administration Review», XXX, pp. 314-325.

- (1972), Four Systems of Policy, Politics, and Choice, in «Public Administration Review», XXXII, pp. 298-310.

- (1985), The State in Politics. The Relation Between Policy and Administration, in R. Noll (a cura di), Regulatory Policy and the Social Sciences, Berkeley, University of California Press, pp. 67-105.

- (1988), An Assessment of Kellow's «Promoting Elegance in Policy Theory», in «Policy Studies Journal», XVI, pp. 725-728.

McKinney, J.C. (1966), Constructive Typology and Social Theory, New York, Appleton Century Crofts.

- (1970), Sociological Theory and the Process of Typification, in J.C. McKinney e E.A. Tiryakian (a cura di), Theoretical Sociology. Perspective and Developments, New York, Meredith Corporation, pp. 235-269.

Malkin, J. e A. Wildavsky (1991), Why the Traditional Distinction Between Public and Private Goods Should Be Abandoned, in «Journal of Theoretical Politics», IV, pp. 355-378.

Marradi, A. (1987), Concetti e metodi per la ricerca sociale, Firenze, La Giuntina, $3^{\mathrm{a}}$ ed.

- (1990), On Classification, in A. Bebler e J. Seroja (a cura di), Contemporary Political Systems. Classifications and Typologies, London, Lynne Rienner, pp. 11-43.

Morlino, L. (1986), Democrazie, in G. Pasquino (a cura di), Manuale di scienza della politica, Bologna, Il Mulino, pp. 83-135.

Olson, M. (1965), The Logic of Collective Action, Cambridge, Harvard University Press; trad. it. La logica dell'azione collettiva, Milano, Feltrinelli, 1983.

Oppenheim, F.E. (1975), The Language of Political Inquiry: Problems of Clarification, in F.I. Greenstein e N.W. Polsby (a cura di), Handbook of Political Science, Reading, Addison Wesley $\mathrm{Pu}-$ blishing Company, vol. I, pp. 283-335; trad. it. Problemi di chiarificazione del linguaggio, in D. Fisichella (a cura di), Metodo scientifico e ricerca politica, Roma, Nis, 1985, pp. 189-239.

Ostrom, V. e E. Ostrom (1978), Public Goods and Public Choices, in E.S. Savas (a cura di), Alternatives for Delivering Public Services: Toward Improved Performance, Boulder, Co, Westview Press, pp. 7-49.

Panebianco, A. (1989), Le scienze sociali e la politica, in A. Panebianco (a cura di), L'analisi della politica, Bologna, Il Mulino, pp. 13-65.

Pennock, J.R. (1979), Another Legislative Typology, in «Journal of Politics», 41, pp. 1206-1213.

Rakoff, S.H. e G.F. Schaefer (1970), Politics Policy, and Political Science: Theoretical Alternatives, in «Politics and Society», I, pp. 51- 77. 
Regonini, G. (1985), Le politiche sociali in Italia: metodi di analisi, in «Rivista italiana di scienza politica», XV, pp. 335-377.

- (1989), Lo studio delle politiche pubbliche, in A. Panebianco (a cura di), L'analisi della politica, Bologna, Il Mulino, pp. 491-516.

Richardson, J.J. et al. (1982), The Concept of Policy Style, in J.J. Richardson (a cura di), Policy Styles in Western Europe, London, Allen \& Unwin, pp. 1-16.

Rose, R. (1969), Comparing Public Policy, in «European Journal of Political Research», I, pp. 67-94.

- (1984), Comparative Policy Analysis: The Programme Approach, Glasgow, Centre for the Study of Public Policy, Studies in Public Policy 138.

Salisbury, R.H. (1968), The Analysis of Public Policy: A Search for Theories and Roles, in A. Ranney (a cura di), Political Science and Public Policy, Chicago, Markham, pp. 151-175.

Salisbury, R.H. e J. Heinz (1970), A Theory of Policy Analysis and Some Preliminary Applications, in I. Sharkansky (a cura di), Policy Analysis in Political Science, Chicago, Markham, pp. 39-60.

Samuelson, P. (1954), The Pure Theory of Public Expenditure, in «Review of Economic and Statistics», XXXVI, pp. 387-390.

Sartori, G. (1979), La politica. Logica e metodo in scienze sociali, Milano, Sugar Co.

Schattschneider, E.E. (1960), The Semi-Sovereign People, Hillsdale, The Driden Press, $2^{a}$ ed. (1975).

Sheffer, G. (1977), Reversibility of Policies and Pattern of Politics, in «Policy Studies Journal, V, pp. 535-554.

Smith, T.A. (1969), Toward a Comparative Theory of the Policy Process, in «Comparative Politics», I, pp. 498-515.

- (1975), The Comparative Policy Process, Santa Barbara, Clio Books.

Spitzer, R.J. (1983a), The Presidency and Public Policy: A Preliminary Inquiry, University, University of Alabama Press.

- (1983b), Presidential Policy Determinism, in «Presidential Studies Quarterly», Fall, pp. 556-574.

- (1987), Promoting Policy Theory: Revising the Arenas of Power, in «Policy Studies Journal», XV, pp. 675-689.

Steinberger, P.J. (1980), Typologies of Public Policies: Meaning Construction and the Policy Process, in «Social Sciences Quarterly», LXI, pp. 185-207

Stigler, G.J. (1971), The Theory of Economic Regulation, in «The Bell Journal of Economic and Management Science», II, pp. 3-21.

Tatalovich, R. e B. Daynes (1984), Moral Controversies and the Policymaking Process, in «Policy Studies Journal», XIII, pp. 207-222.

Tiryakian, E.A. (1968), Typologies, in D.L. Sills (a cura di), International Encyclopedia of the Social Sciences, New York, Macmillan, vol. 15. 
Uslaner, E.M. (1985), Energy, Issue, and Policy Typologies, in H.M. Ingram (a cura di), Public Policy and the Natural Environment, Greenwich, JAI Press, pp. 101-122.

Weber, M. (1922a), Gesammelte Aufsatze zur Wissenschaftslebere, Tubingen, Mohr; trad. it., Il metodo nelle scienze storico-sociali, Milano, Mondadori, 1980.

- (1922b), Wirtschaft und Gesellschaft, Tubingen, Mohr; trad. it., Economia e Società, Milano, Comunità, 1980, 5 voll.

Wilson, J.Q. (1973), Political Organizations, New York, Basic Books.

- (1974), The Politics of Regulation, in McKie J.W. (a cura di), Social Responsability and the Business Predicament, Washington D.C., The Brookings Institution, pp. 135-164.

- (1980), The Politics of Regulation, in J.Q. Wilson (a cura di), The Politics of Regulation, New York, Basic Books, pp. 357-394. 